Turk. J. Math. Comput. Sci.

13(2)(2021) 294-309

(C) MatDer

DOI : $10.47000 /$ tjmcs.XX

\title{
Neutrosophic Extension of N-soft Set and Similarity-Based Decision-Making
}

\author{
Naime Demirtaş ${ }^{1}$ (i), Orhan Dalkiliç ${ }^{1, *}$ (i) \\ ${ }^{1}$ Department of Mathematics, Faculty of Arts and Sciences, Mersin University, 33433, Yenişehir/Mersin, Turkey.
}

Received: 30-04-2021 • Accepted: 25-11-2021

\begin{abstract}
AвstRACt. In this paper, the extension of $\mathrm{N}$-soft sets, which is a very important mathematical model in non-binary evaluations to overcome uncertainty, under neutrosophic logic are studied and neutrosophic N-soft sets are introduced and are motivated. This new mathematical model, which deals with neutrosophic logic and N-soft set, which have been studied extensively in recent years to overcome uncertainty, aims to express the uncertainty situations encountered in the best way and thus approach the ideal in decision making. Moreover, some fundamental properties, products and useful operations are given for this new mathematical model. Then, we defined distance measures between two neutrosophic N-soft sets and expressed similarity measures based on decision making problem. Finally, an application is given that illustrates how uncertainty situations can be expressed in a decision-making problem by using the suggested similarity measures.
\end{abstract}

2010 AMS Classification: 03E72, 08A72

Keywords: Neutrosophic N-soft set, distance measures, similarity measures, decision making.

\section{INTRODUCTION}

Many problems in areas such as medical, social, economic, environmental, where the human factor is effective, involve uncertainty imprecision, or subjectivity. That's why, many researchers have worked on different mathematical models to express and solve this type of problems. The first of the mathematical models put forward to express the uncertainty problems correctly is the fuzzy set (briefly FS) theory put forward by Zadeh [52] in 1965. Indeed, although many other mathematical models such as rough set theory [42], intuitionistic fuzzy set theory [10] and neutrosophic set theory [49] have been introduced in the literature since 1965, yet the soft set (briefly SS) theory introduced by Molodsov [41] in 1999 is strong enough, which leads all models presented so far to deal with uncertainty. The reason why this set theory proposed by Molodsov against uncertainty is so powerful is that it can eliminate the deficiency of a parameterization tool which is not present in other set theories and is very convenient in expressing the decision-making process. Thanks to its success in expressing the uncertainty problems encountered, this set theory has been easily applied to many areas such as theory of measurement, smoothness of functions, Riemann Integration and game theory. The application area and diversity of the soft set theory brought to the literature by Molodsov is rapidly increasing due to its success in expressing uncertainty $[8,9,18,20,24-26,35]$. To give a few examples, Maji et al. [36,37] first defined concepts of complement, subset, equality, absolute SS and null SS for SSs. In addition, they proposed an application of SSs to solve the uncertainty problems encountered in a near ideal way. Chen et al. [16] and Kong et al. [34] worked on parameter reduction and normal parameter reduction methods for SSs, respectively. Then, Ali et al. [7] defined some new operations between SSs and Qin and Hong [43] defined concept of soft equality. Çağman and Enginoğlu [17]

*Corresponding Author

Email addresses: naimedemirtas@mersin.edu.tr (N. Demirtaş), orhandlk952495@hotmail.com (O. Dalkılıç) 
studied on products of SSs by redefining the operations between the two SSs. Especially in recent years, interest in SS theory has been increased greatly, and many interesting applications of this theory have been expanded by embedding the ideas of mathematical models such as FS (e.g. [19, 20, 28]), rough set (e.g. [25, 29]), intuitionistic fuzzy set (e.g. [32,38]), neutrosophic set (briefly NS) (e.g. [39,40]).

Another mathematical model introduced to the literature as a result of the effort to express uncertainty situations in an ideal way is the neutrosophic set theory proposed by Smarandache [47]. Obviously; the concept of neutrosophy, a branch of philosophy that studies the nature, origin and scope of neutralities, as well as their interactions with different ideational spectra, was influential in the construction of this theory. Neutrosophy considers a theory, concept, proposition, event, or entity $\langle A\rangle$ in relation to its opposite $\langle$ antiA $>$, and with their neutral $<$ neut $A\rangle$. Moreover, neutrosophy is the basis of neutrosophic statistics, neutrosophic probability, neutrosophic logic (briefly NL) and NS. Here, NL is a general framework for unification of many logics, such as paraconsistent logic, fuzzy logic, intuitionistic logic, intuitionistic fuzzy logic etc. NL aims is to characterize each logical statement in a 3D-neutrosophic space, where each dimension of the space represents respectively the truth (T), the falsehood (F), and the indeterminacy (I) of the statement under consideration, where T, I, F are not necessarily any connection between them. The articles [47-49,51] can be examined for more detailed information on this subject. After the NL and consequently the NS theory by considering a truth membership function, an indeterminacy membership function and a falsity membership function was suggested by Smarandache, it was thought that it could be used as an effective tool in the fight against uncertainty by many researchers. One of the most important reasons for this is that NSs are a generalization of mathematical models such as classical sets, conventional FS [52], interval-valued fuzzy set [30,31,46,53], intuitionistic fuzzy set [10, 21], interval-valued intuitionistic fuzzy set [11]. In other words, NS generalizes the existing ideas. For example; the NS handles indeterminate data whereas FS and intuitionistic fuzzy set failed when the relation is indeterminate. Single valued neutrosophic set was developed by Smarandache [47] and Wang et al. [51] in order to easily express uncertainty problems encountered in fields such as engineering and scientific in the following years. Then, neutrosophic soft set [40] have been brought to the literature by combining NS and SS theories, which are very successful in expressing uncertainty problems. In this way, studies on the neutrosophic soft sets have begun to be examined more by many researchers (e.g. $[1,12,13,45,50])$. Some more literature on neutrosophy, NL, NS, neutrosophic soft set can be found in $[14,15,22,23,33]$.

SS theory provide binary evaluation of the objects and mathematical models such as FSs, intuitionistic fuzzy sets and NSs associate values in the interval $[0,1]$. However, these mathematical models fail to associate non-binary evaluations of uncertainty problems encountered. These evaluations are expected in ranking or rating positions. The ranking can be expressed in multinary values such as grades, number of stars, dots. In 2018, Fatimah et al. [27] proposed N-soft set (briefly N-SS) theory, which is an extended mathematical model of SSs, for non-binary evaluations encountered in uncertainty problems. They also gave some properties and algebraic definitions for the proposed theory. Moreover, they gave some examples that prove that N-SSs are a convincing mathematical model for binary and non-binary evaluations in decision-making problems, and proposed some decision-making algorithm based on N-SSs. After the N-SSs were introduced into the literature, many studies were carried out to solve the decision making problems in set types such as hesitant $\mathrm{N}$-soft set [3], fuzzy N-soft set [2], intuitionistic fuzzy $\mathrm{N}$-soft rough sets [6], hesitant fuzzy N-soft set [4], N-soft topology [44], interval-valued hesitant fuzzy N-soft set [5], and so on.

We mentioned above that researchers have developed many hybrid set models, considering that the uncertainty situations encountered in daily life can be quite complex for N-SSs. In this paper, we examined the extension of N-SSs with NL and introduced the concept of neutrosophic N-soft set (briefly NN-SS). In other words, the theory of this new concept was developed by examining the N-SS theory under NL and propose a decision support technique with the help of similarity measurements based on distance measurements.

The organization of this paper is as follows. In the second section, some mathematical models for the construction of N-SSs are reminded and the general construction of the proposed theory is mentioned. In the third section, some algebraic properties and basic operations of NN-SSs are introduced. The fourth section describes similarity measure based on the distance measures of NN-SSs. The fifth section is devoted to a method for the decision-making problem 
by means of the similarity measures between NN-SSs. The final section consists of the conclusion of the paper.

Throughout this study, let $\mathcal{E}=\left\{x_{1}, x_{2}, \ldots, x_{n}\right\}$ be a set of parameters and $\mathcal{U}=\left\{u_{1}, u_{2}, \ldots, u_{m}\right\}$ be a universe of objects. Also, let $|\mathcal{U}|$ be the number of objects in $\mathcal{U}$ and $2^{\mathcal{U}}$ denote the power set of $\mathcal{U}$.

\section{Preliminaries}

In this section, we define the NN-SS. For this, firstly let's remind the concepts of SS and N-SS.

Molodtsov [41] defined SS in the following way,

Definition 2.1. [41] A pair $(\mathcal{F}, \mathcal{E})$ is called a SS over $\mathcal{U}$, where $\mathcal{F}$ is a mapping given by $\mathcal{F}: \mathcal{E} \rightarrow 2^{\mathcal{U}}$. In other words, a SS over $\mathcal{U}$ is a parameterized family of subsets of $\mathcal{U}$ for $x \in \mathcal{E}, \mathcal{F}(x)$ may be considered as the set of $x$-approximate elements of $(\mathcal{F}, \mathcal{E})$.

Let's recall the N-SS theory, an extended mathematical model of SSs proposed by Fatimah et al. [27]. The most important advantage of this set theory is that it is a convincing mathematical model for binary and non-binary evaluations encountered in uncertainty problems. The definition of N-SSs given by Fatimah et al. [27] is as follows:

Definition 2.2. [27] Let $\mathcal{R}=\{0,1,2, \ldots, N-1\}$ be a collection of ordered grades, where $N=\{2,3, \ldots\}$. Then, the triplet $(\widehat{F}, \mathcal{E}, N)$ is called an N-SS on $\mathcal{U}$ if $\widehat{F}$ is a mapping $\widehat{F}: \mathcal{E} \rightarrow 2^{\mathcal{U} \times \mathcal{R}}$ where for each $x \in \mathcal{E}$,

$$
\widehat{F}(x)=\left\{\left(u_{k}, \wp_{\mathcal{E}_{\overparen{F}}}^{k}\right): u_{k} \in \mathcal{U} \text { and } \wp_{\mathcal{E}_{\overparen{F}}}^{k} \in \mathcal{R} \text { is the grade of } u_{k}\right\}
$$

such that there is a unique grade $\wp_{\mathcal{E}_{\widehat{F}}}^{k}$ for each $u_{k} \in \mathcal{U}$.

Now, we define the NN-SS as follows:

Definition 2.3. Let $\mathcal{K}=\langle T, I, F\rangle=\{\langle t, i, f\rangle: t \in T, i \in I, f \in F\}$ be a set of ordered neutrosophic grades, where $T=$ $\{0,1,2, \ldots, N-1\}=I=F$, denote grades of truth membership information, indeterminacy-membership information and falsity-membership information for $N=\{2,3, \ldots\}$. A triplet $(\Gamma, \mathcal{E}, N)$ is called a NN-SS over $\mathcal{U}$ if $\Gamma$ is a mapping $\Gamma: \mathcal{E} \rightarrow 2^{\mathcal{U}_{N} \times \mathcal{K}}$ where for each $x \in \mathcal{E}$ and $\mathcal{U}_{N}=\mathcal{U} \times N$,

$$
\Gamma(x)=\left\{\left(u_{k},\left\langle t_{\Gamma_{x}}^{k}, i_{\Gamma_{x}}^{k}, f_{\Gamma_{x}}^{k}\right\rangle\right): u_{k} \in \mathcal{U} \text { and }\left\langle t_{\Gamma_{x}}^{k}, i_{\Gamma_{x}}^{k}, f_{\Gamma_{x}}^{k}\right\rangle \in \mathcal{K} \text { is neutrosophic grade of } u_{k}\right\}
$$

such that

$$
(\Gamma, \mathcal{E}, N)=\left\{\left(x_{l},\left(u_{k},\left\langle t_{\Gamma_{x}}^{k}, i_{\Gamma_{x}}^{k}, f_{\Gamma_{x}}^{k}\right\rangle\right): u_{k} \in \mathcal{U}\right): x_{l} \in \mathcal{E}\right\}
$$

there is a unique neutrosophic grade $\left\langle t_{\Gamma_{x}}^{k}, i_{\Gamma_{x}}^{k}, f_{\Gamma_{x}}^{k}\right\rangle$ for each $u_{k} \in \mathcal{U}$.

State that the set of all NN-SSs on $\mathcal{U}$ is denoted by $N N-S S(\mathcal{U})$.

Definition 2.4. Let $(\Gamma, \mathcal{E}, N) \in N N-S S(\mathcal{U})$ Then the neutrosophic N-soft matrix for the NN-SS $(\Gamma, \mathcal{E}, N)$ is $\mathbf{M}_{\Gamma}$, where

$$
\mathbf{M}_{\Gamma}=\left(\begin{array}{cccc}
\Lambda_{11}^{\Gamma} & \Lambda_{12}^{\Gamma} & \ldots & \Lambda_{1 n}^{\Gamma} \\
\Lambda_{21}^{\Gamma} & \Lambda_{22}^{\Gamma} & \ldots & \Lambda_{2 n}^{\Gamma} \\
\vdots & \vdots & \ddots & \vdots \\
\Lambda_{m 1}^{\Gamma} & \Lambda_{m 2}^{\Gamma} & \ldots & \Lambda_{m n}^{\Gamma}
\end{array}\right)_{m \times n} .
$$

Each component in the expressed neutrosophic $\mathrm{N}$-soft matrix for $(\Gamma, \mathcal{E}, N)$ is made up of triples in the form of $\Lambda_{k l}^{\Gamma}=$ $\left\langle t_{\Gamma_{x_{l}}}^{k}, i_{\Gamma_{x_{l}}}^{k}, f_{\Gamma_{x_{l}}}^{k}\right\rangle$ for $1 \leq k \leq m$ and $1 \leq l \leq n$.

Example 2.5. Let's say that a person is considering buying a house. Also, he/she determines the parameters as $\mathcal{E}=\left\{x_{1}\right.$ : Luxurious, $x_{2}:$ Economic, $x_{3}$ : Large garden, $x_{4}:$ Comfortable $\}$. The person who examines the houses owned by the realtor determines three houses as a result of the preliminary evaluation. Their opinions about these houses are expressed as follows with the help of the N5-SS $(\Gamma, \mathcal{E}, 5)$.

$$
(\Gamma, \mathcal{E}, 5)=\left\{\begin{array}{c}
\left(x_{1},\left(u_{1},\langle 4,2,3\rangle\right),\left(u_{2},\langle 4,1,2\rangle\right),\left(u_{3},\langle 3,2,0\rangle\right),\left(u_{4},\langle 1,3,2\rangle\right)\right), \\
\left(x_{2},\left(u_{1},\langle 0,2,4\rangle\right),\left(u_{2},\langle 3,1,4\rangle\right),\left(u_{3},\langle 2,4,1\rangle\right),\left(u_{4},\langle 2,4,1\rangle\right)\right), \\
\left(x_{3},\left(u_{1},\langle 3,4,2\rangle\right),\left(u_{2},\langle 4,3,1\rangle\right),\left(u_{3},\langle 2,3,4\rangle\right),\left(u_{4},\langle 3,3,2\rangle\right)\right)
\end{array}\right\} .
$$


Here, the ordered triple $\langle 2,4,1\rangle$ means the truth-membership (information) grade, indeterminacy-membership (information) grade and falsity-membership (information) grade of the house $u_{3}$ with respect to the parameter $x_{2}$ : Economic.

The opinions given in $(\Gamma, \mathcal{E}, 5)$ can be expressed more clearly with $\mathbf{M}_{\boldsymbol{\Gamma}}$. The matrix representation of the N5-SS $(\Gamma, \mathcal{E}, 5)$ is given as follows:

$$
\mathbf{M}_{\boldsymbol{\Gamma}}=\left(\begin{array}{ccc}
\langle 4,2,3\rangle & \langle 0,2,4\rangle & \langle 3,4,2\rangle \\
\langle 4,1,2\rangle & \langle 3,1,4\rangle & \langle 4,3,1\rangle \\
\langle 3,2,0\rangle & \langle 2,4,1\rangle & \langle 2,3,4\rangle \\
\langle 1,3,2\rangle & \langle 2,4,1\rangle & \langle 3,3,2\rangle
\end{array}\right) .
$$

Unless otherwise noted in the definitions, properties and remarks to be given hereafter, it will be accepted as $(\Gamma, \mathcal{E}, 5) \in N N-S S(\mathcal{U})$ and $\langle T, I, F\rangle$ such that $T=\{0,1,2, \ldots, N-1\}=I=F$.

Definition 2.6. A null NN-SS on $\mathcal{U}$ is defined by $\left(\Gamma_{\varnothing}, \mathcal{E}, N\right)$, where $\Gamma_{\varnothing}: \mathcal{E} \rightarrow 2^{\mathcal{U}_{N} \times\langle 0, N, N\rangle}$ with property $\Gamma_{\varnothing}(x)=$ $\left\{\left(u_{k},\langle 0, N-1, N-1\rangle\right): \forall u_{k} \in \mathcal{U}\right\}$ for each $x \in \mathcal{E}$.

Definition 2.7. A absolute NN-SS on $\mathcal{U}$ is defined by $\left(\Gamma_{\mathcal{U}}, \mathcal{E}, N\right)$, where $\Gamma_{\mathcal{U}}: \mathcal{E} \rightarrow 2^{\mathcal{U}_{N} \times\langle N, 0,0\rangle}$ with property $\Gamma_{\mathcal{U}}(x)=$ $\left\{\left(u_{k},\langle N-1,0,0\rangle\right): \forall u_{k} \in \mathcal{U}\right\}$ for each $x \in \mathcal{E}$.

Example 2.8. Let $\mathcal{U}=\left\{u_{1}, u_{2}, u_{3}\right\}$ be the set of three cars under consideration, $\mathcal{E}=\left\{x_{1}:\right.$ Fuel efficient, $x_{2}$ : Costly, $x_{3}$ : Luxurious $\}$ be the set of parameters, then the null N8-SS and the absolute N8-SS defined on $\mathcal{U}$ are given in the matrix representation $\mathbf{M}_{\mathbf{\Gamma}_{\varnothing}}$ and $\mathbf{M}_{\boldsymbol{\Gamma}_{\mathcal{U}}}$, respectively,

$$
\begin{aligned}
\mathbf{M}_{\Gamma_{\varnothing}} & =\left(\begin{array}{lll}
\langle 0,7,7\rangle & \langle 0,7,7\rangle & \langle 0,7,7\rangle \\
\langle 0,7,7\rangle & \langle 0,7,7\rangle & \langle 0,7,7\rangle \\
\langle 0,7,7\rangle & \langle 0,7,7\rangle & \langle 0,7,7\rangle
\end{array}\right), \\
\mathbf{M}_{\Gamma_{\mathcal{U}}} & =\left(\begin{array}{lll}
\langle 7,0,0\rangle & \langle 7,0,0\rangle & \langle 7,0,0\rangle \\
\langle 7,0,0\rangle & \langle 7,0,0\rangle & \langle 7,0,0\rangle \\
\langle 7,0,0\rangle & \langle 7,0,0\rangle & \langle 7,0,0\rangle
\end{array}\right) .
\end{aligned}
$$

Definition 2.9. $\left(\Gamma_{1}, \mathcal{E}, N\right)$ is neutrosophic $N$-soft subset of $\left(\Gamma_{2}, \mathcal{E}, N\right)$ if for every $x \in \mathcal{E}, t_{\Gamma_{1_{x}}}^{k} \leq t_{\Gamma_{2_{x}}}^{k}, i_{\Gamma_{1_{x}}}^{k} \geq i_{\Gamma_{2 x}}^{k}$ and $f_{\Gamma_{1_{x}}}^{k} \geq f_{\Gamma_{2_{x}}}^{k}$ for all $u_{k} \in \mathcal{U}$, where $\left\langle t_{\Gamma_{1_{x}}}^{k}, i_{\Gamma_{1_{x}}}^{k}, f_{\Gamma_{1_{x}}}^{k}\right\rangle$ and $\left\langle t_{\Gamma_{2_{x}}}^{k}, i_{\Gamma_{2 x}}^{k}, f_{\Gamma_{2_{x}}}^{k}\right\rangle$ represent the neutrosophic grades of $\Gamma_{1}(x)$ and $\Gamma_{2}(x)$, respectively. It is symbolized by $\left(\Gamma_{1}, \mathcal{E}, N\right) \sqsubseteq\left(\Gamma_{2}, \mathcal{E}, N\right)$.

Definition 2.10. $\left(\Gamma_{1}, \mathcal{E}, N\right)$ and $\left(\Gamma_{2}, \mathcal{E}, N\right)$ are equal NN-SSs if for every $x \in \mathcal{E}, t_{\Gamma_{1_{x}}}^{k}=t_{\Gamma_{2_{x}}}^{k}, i_{\Gamma_{1_{x}}}^{k}=i_{\Gamma_{2_{x}}}^{k}$ and $f_{\Gamma_{1_{x}}}^{k}=f_{\Gamma_{2_{x}}}^{k}$ for all $u_{k} \in \mathcal{U}$, where $\left\langle t_{\Gamma_{1 x}}^{k}, i_{\Gamma_{1_{x}}}^{k}, f_{\Gamma_{1_{x}}}^{k}\right\rangle$ and $\left\langle t_{\Gamma_{2 x}}^{k}, i_{\Gamma_{2 x}}^{k}, f_{\Gamma_{2 x}}^{k}\right\rangle$ represent the neutrosophic grades of $\Gamma_{1}(x)$ and $\Gamma_{2}(x)$, respectively. It is symbolized by $\left(\Gamma_{1}, \mathcal{E}, N\right)=\left(\Gamma_{2}, \mathcal{E}, N\right)$.

Example 2.11. Let $\mathcal{U}=\left\{u_{1}, u_{2}, u_{3}\right\}$ be an universe and $\mathcal{E}=\left\{x_{1}, x_{2}, x_{3}, x_{4}\right\}$ be a set of parameters. Consider the N8-SSs on $\mathcal{U}$ as given in the matrix representation $\mathbf{M}_{\Gamma_{1}}$ and $\mathbf{M}_{\Gamma_{2}}$. It is clear that $\left(\Gamma_{1}, \mathcal{E}, 8\right) \sqsubseteq\left(\Gamma_{2}, \mathcal{E}, 8\right)$.

$$
\begin{aligned}
\mathbf{M}_{\Gamma_{1}} & =\left(\begin{array}{llll}
\langle 5,2,7\rangle & \langle 3,5,6\rangle & \langle 4,1,4\rangle & \langle 5,4,6\rangle \\
\langle 4,3,6\rangle & \langle 2,6,1\rangle & \langle 3,3,2\rangle & \langle 3,5,3\rangle \\
\langle 6,1,4\rangle & \langle 4,6,3\rangle & \langle 5,6,3\rangle & \langle 1,4,4\rangle
\end{array}\right), \\
\mathbf{M}_{\Gamma_{2}} & =\left(\begin{array}{llll}
\langle 6,1,5\rangle & \langle 4,3,3\rangle & \langle 6,0,3\rangle & \langle 6,2,4\rangle \\
\langle 7,2,3\rangle & \langle 3,2,0\rangle & \langle 4,2,1\rangle & \langle 4,3,1\rangle \\
\langle 7,0,2\rangle & \langle 5,4,2\rangle & \langle 6,3,2\rangle & \langle 5,2,3\rangle
\end{array}\right) .
\end{aligned}
$$

Proposition 2.12. Let $(\Gamma, \mathcal{E}, N),\left(\Gamma_{1}, \mathcal{E}, N\right),\left(\Gamma_{2}, \mathcal{E}, N\right),\left(\Gamma_{3}, \mathcal{E}, N\right) \in N N-S S(\mathcal{U})$. Then, i. $\left(\Gamma_{\varnothing}, \mathcal{E}, N\right) \sqsubseteq(\Gamma, \mathcal{E}, N) \sqsubseteq\left(\Gamma_{\mathcal{U}}, \mathcal{E}, N\right)$. ii. If $\left(\Gamma_{1}, \mathcal{E}, N\right) \sqsubseteq\left(\Gamma_{2}, \mathcal{E}, N\right)$ and $\left(\Gamma_{2}, \mathcal{E}, N\right) \sqsubseteq\left(\Gamma_{3}, \mathcal{E}, N\right)$, then $\left(\Gamma_{1}, \mathcal{E}, N\right) \sqsubseteq\left(\Gamma_{3}, \mathcal{E}, N\right)$. iii. If $\left(\Gamma_{1}, \mathcal{E}, N\right)=\left(\Gamma_{2}, \mathcal{E}, N\right)$ and $\left(\Gamma_{2}, \mathcal{E}, N\right)=\left(\Gamma_{3}, \mathcal{E}, N\right)$, then $\left(\Gamma_{1}, \mathcal{E}, N\right)=\left(\Gamma_{3}, \mathcal{E}, N\right)$.

Proof. It is clear from Definitions 2.6, 2.7, 2.9 and 2.10. 


\section{Some Basic Operations on NN-SSs}

In this section, we describe some basic operations on the NN-SSs.

Definition 3.1. A complement of $\mathrm{NN}-\mathrm{SS}(\Gamma, \mathcal{E}, N)$ is defined by $(\Gamma, \mathcal{E}, N)^{c}=\left(\Gamma^{c}, \mathcal{E}, N\right)$, where

$$
\Gamma^{c}(x)=\left\{\left(u_{k}:\left\langle t_{\Gamma_{x}^{c}}^{k}, i_{\Gamma_{x}^{c}}^{k}, f_{\Gamma_{x}^{c}}^{k}\right\rangle\right): u_{k} \in \mathcal{U}\right\}
$$

for every $x \in \mathcal{E}$ such that $t_{\Gamma_{x}^{c}}^{k}=f_{\Gamma_{x}}^{k}, i_{\Gamma_{x}^{c}}^{k}=(N-1)-i_{\Gamma_{x}}^{k}$ and $f_{\Gamma_{x}^{c}}^{k}=t_{\Gamma_{x}}^{k}$ for all $u_{k} \in \mathcal{U}$.

Example 3.2. Consider the N5-SS $(\Gamma, \mathcal{E}, 5)$ as given in Example 2.5. Then its complement is given in $\mathbf{M}_{\Gamma^{\mathbf{c}}}$.

$$
\mathbf{M}_{\Gamma^{\mathbf{c}}}=\left(\begin{array}{ccc}
\langle 3,2,4\rangle & \langle 4,2,0\rangle & \langle 2,0,3\rangle \\
\langle 2,3,4\rangle & \langle 4,3,3\rangle & \langle 1,1,4\rangle \\
\langle 0,2,3\rangle & \langle 1,0,2\rangle & \langle 4,1,2\rangle \\
\langle 2,1,1\rangle & \langle 1,0,2\rangle & \langle 2,1,3\rangle
\end{array}\right) .
$$

Proposition 3.3. Let $(\Gamma, \mathcal{E}, N),\left(\Gamma_{1}, \mathcal{E}, N\right),\left(\Gamma_{2}, \mathcal{E}, N\right) \in N N-S S(\mathcal{U})$. Then,

i. $\left(\Gamma_{\varnothing}, \mathcal{E}, N\right)^{c}=\left(\Gamma_{\mathcal{U}}, \mathcal{E}, N\right)$ and $\left(\Gamma_{\mathcal{U}}, \mathcal{E}, N\right)^{c}=\left(\Gamma_{\varnothing}, \mathcal{E}, N\right)$.

ii. $\left(\Gamma^{c}, \mathcal{E}, N\right)^{c}=\left((\Gamma, \mathcal{E}, N)^{c}\right)^{c}=(\Gamma, \mathcal{E}, N)$.

iii. If $\left(\Gamma_{1}, \mathcal{E}, N\right) \sqsubseteq\left(\Gamma_{2}, \mathcal{E}, N\right)$, then $\left(\Gamma_{2}, \mathcal{E}, N\right)^{c} \sqsubseteq\left(\Gamma_{1}, \mathcal{E}, N\right)^{c}$.

Proof. It is clear from Definitions 2.9 and 3.1.

Definition 3.4. Other types of complement are as follows:

$i$. The weak complement of NN-SS $(\Gamma, \mathcal{E}, N)$ is defined by $(\Gamma, \mathcal{E}, N)^{w}=\left(\Gamma^{w}, \mathcal{E}, N\right)$, where

$$
\Gamma^{w}(x)=\left\{\left(u_{k}:\left\langle t_{\Gamma_{x}^{w}}^{k}, i_{\Gamma_{x}^{w}}^{k}, f_{\Gamma_{x}^{w}}^{k}\right\rangle\right): u_{k} \in \mathcal{U}\right\}
$$

for every $x \in \mathcal{E}$ such that $t_{\Gamma_{x}^{w}}^{k} \neq t_{\Gamma_{x}}^{k}, i_{\Gamma_{x}^{w}}^{k} \neq i_{\Gamma_{x}}^{k}$ and $f_{\Gamma_{x}^{w}}^{k} \neq f_{\Gamma_{x}}^{k}$ for all $u_{k} \in \mathcal{U}$.

ii. The top weak complement of NN-SS $(\Gamma, \mathcal{E}, N)$ is defined by $(\Gamma, \mathcal{E}, N)^{t w}=\left(\Gamma^{t w}, \mathcal{E}, N\right)$, where

$$
\Gamma^{t w}(x)=\left\{\left(u_{k}:\left\langle t_{\Gamma_{x}^{t w}}^{k}, i_{\Gamma_{x}^{t w}}^{k}, f_{\Gamma_{x}^{t w}}^{k}\right\rangle\right): u_{k} \in \mathcal{U}\right\}
$$

for every $x \in \mathcal{E}$ such that

$$
\begin{gathered}
t_{\Gamma_{x}^{t w}}^{k}=\left\{\begin{array}{ll}
0 & \text { if } t_{\Gamma_{x}}^{k}=N-1 \\
N-1 & \text { otherwise }
\end{array},\right. \\
i_{\Gamma_{x}^{t w}}^{k}=\left\{\begin{array}{ll}
N-1 & \text { if } i_{\Gamma_{x}}^{k}=0 \\
0 & \text { otherwise }
\end{array},\right.
\end{gathered}
$$

and

$$
f_{\Gamma_{x}^{t w}}^{k}= \begin{cases}N-1 & \text { if } f_{\Gamma_{x}}^{k}=0 \\ 0 & \text { otherwise }\end{cases}
$$

for all $u_{k} \in \mathcal{U}$.

iii. The bottom weak complement of NN-SS $(\Gamma, \mathcal{E}, N)$ is defined by $(\Gamma, \mathcal{E}, N)^{b w}=\left(\Gamma^{b w}, \mathcal{E}, N\right)$, where

$$
\Gamma^{b w}(x)=\left\{\left(u_{k}:\left\langle t_{\Gamma_{x}^{b w}}^{k}, i_{\Gamma_{x}^{b w}}^{k}, f_{\Gamma_{x}^{b w}}^{k}\right\rangle\right): u_{k} \in \mathcal{U}\right\}
$$

for every $x \in \mathcal{E}$ such that

$$
\begin{gathered}
t_{\Gamma_{x}^{b w}}^{k}=\left\{\begin{array}{ll}
N-1 & \text { if } t_{\Gamma_{x}}^{k}=0 \\
0 & \text { otherwise }
\end{array},\right. \\
i_{\Gamma_{x}^{b w}}^{k}=\left\{\begin{array}{ll}
0 & \text { if } i_{\Gamma_{x}}^{k}=N-1 \\
N-1 & \text { otherwise }
\end{array},\right.
\end{gathered}
$$

and

$$
f_{\Gamma_{x}^{b w}}^{k}= \begin{cases}0 & \text { if } f_{\Gamma_{x}}^{k}=N-1 \\ N-1 & \text { otherwise }\end{cases}
$$

for all $u_{k} \in \mathcal{U}$. 
Example 3.5. Consider the N5-SS $(\Gamma, \mathcal{E}, 5)$ as given in Example 2.5. Then their week complement, top weak complement and bottom weak complement are given in $\mathbf{M}_{\Gamma^{\mathrm{w}}}, \mathbf{M}_{\Gamma^{\mathrm{tw}}}$ and $\mathbf{M}_{\Gamma^{\mathrm{b}} \mathbf{w}}$ respectively,

$$
\begin{aligned}
\mathbf{M}_{\Gamma^{w}} & =\left(\begin{array}{lll}
\langle 3,1,4\rangle & \langle 4,3,0\rangle & \langle 2,0,3\rangle \\
\langle 2,3,4\rangle & \langle 2,3,2\rangle & \langle 1,0,4\rangle \\
\langle 0,1,3\rangle & \langle 3,0,2\rangle & \langle 4,1,2\rangle \\
\langle 2,1,1\rangle & \langle 1,0,2\rangle & \langle 2,1,3\rangle
\end{array}\right), \\
\mathbf{M}_{\Gamma^{\text {tw }}} & =\left(\begin{array}{lll}
\langle 0,0,0\rangle & \langle 4,0,0\rangle & \langle 4,0,0\rangle \\
\langle 0,0,0\rangle & \langle 4,0,0\rangle & \langle 0,0,0\rangle \\
\langle 4,0,4\rangle & \langle 4,0,0\rangle & \langle 4,0,0\rangle \\
\langle 4,0,0\rangle & \langle 4,0,0\rangle & \langle 4,0,0\rangle
\end{array}\right), \\
\mathbf{M}_{\Gamma^{b w}} & =\left(\begin{array}{lll}
\langle 0,4,4\rangle & \langle 4,4,0\rangle & \langle 0,0,4\rangle \\
\langle 0,4,4\rangle & \langle 0,4,0\rangle & \langle 0,4,4\rangle \\
\langle 0,4,4\rangle & \langle 0,0,4\rangle & \langle 0,4,0\rangle \\
\langle 0,4,4\rangle & \langle 0,0,4\rangle & \langle 0,4,4\rangle
\end{array}\right) .
\end{aligned}
$$

Proposition 3.6. Let $(\Gamma, \mathcal{E}, N),\left(\Gamma_{1}, \mathcal{E}, N\right),\left(\Gamma_{2}, \mathcal{E}, N\right) \in N N-S S(\mathcal{U})$. Then, for $j=t w, b w$

i. $\left(\Gamma_{\varnothing}, \mathcal{E}, N\right)^{j}=\left(\Gamma_{\mathcal{U}}, \mathcal{E}, N\right)$ and $\left(\Gamma_{\mathcal{U}}, \mathcal{E}, N\right)^{j}=\left(\Gamma_{\varnothing}, \mathcal{E}, N\right)$.

ii. If $\left(\Gamma_{1}, \mathcal{E}, N\right) \sqsubseteq\left(\Gamma_{2}, \mathcal{E}, N\right)$, then $\left(\Gamma_{2}, \mathcal{E}, N\right)^{j} \sqsubseteq\left(\Gamma_{1}, \mathcal{E}, N\right)^{j}$.

iii. $\left((\Gamma, \mathcal{E}, N)^{j}\right)^{w t}=\left((\Gamma, \mathcal{E}, N)^{j}\right)^{w b}=\left((\Gamma, \mathcal{E}, N)^{j}\right)^{c}$.

$i v \cdot\left(\left((\Gamma, \mathcal{E}, N)^{j}\right)^{m}\right)^{l}=(\Gamma, \mathcal{E}, N)^{j}$ for $m=t w, b w, c$ and $l=t w, b w, c$.

Proof. The proof of $i$. and ii. follows from Definitions 2.9, 3.1 and 3.4.

iii. Assume that $j=t w$, then the value of $t_{\Gamma_{x}^{\prime w}}^{k}$ is either $N-1$ or 0 . If $t_{\Gamma_{x}^{w w}}^{k}=N-1$, then $t_{\left(\Gamma^{w w}\right)_{x}^{w w}}^{k}=t_{\left(\Gamma^{w w}\right)_{x}^{b b}}^{k}=t_{\left(\Gamma^{w w}\right)_{x}^{c}}^{k}=0$. On the other hand, if $t_{\Gamma_{x}^{\prime w}}^{k}=0$, then $t_{\left(\Gamma^{w}\right)_{x}^{w w}}^{k}=t_{\left(\Gamma^{w w}\right)_{x}^{b b}}^{k}=t_{\left(\Gamma^{w w}\right)_{x}^{c}}^{k}=N-1$. By adopting the same way, we can complete the case for $j_{\Gamma_{x}^{\prime w}}^{k}$ and $f_{\Gamma_{x}^{\prime w}}^{k}$. Similarly, we can check for the remaining part for $j=b w$ and hence the proof of iii..

iv. Straightforward.

Definition 3.7. An addition of NN-SS $\left(\Gamma_{1}, \mathcal{E}, N\right)$ and $\left(\Gamma_{2}, \mathcal{E}, N\right)$ is defined by $\left(\Gamma_{1}, \mathcal{E}, N\right) \oplus\left(\Gamma_{2}, \mathcal{E}, N\right)=\left(\Gamma_{1} \oplus \Gamma_{2}, \mathcal{E}, N\right)$, where $\left(\Gamma_{1} \oplus \Gamma_{2}\right)(x)=\left\{\left(u_{k}:\left\langle t_{\left(\Gamma_{1} \oplus \Gamma_{2}\right)_{x}}^{k}, i_{\left(\Gamma_{1} \oplus \Gamma_{2}\right)_{x}}^{k}, f_{\left(\Gamma_{1} \oplus \Gamma_{2}\right)_{x}}^{k}\right\rangle\right): u_{k} \in \mathcal{U}\right\}$ for every $x \in \mathcal{E}$ such that

$$
\begin{aligned}
& t_{\left(\Gamma_{1} \oplus \Gamma_{2}\right)_{x}}^{k}= \begin{cases}t_{\Gamma_{1 x}}^{k}+t_{\Gamma_{2 x}}^{k} & \text { if } 0 \leq t_{\Gamma_{1 x}}^{k}+t_{\Gamma_{2 x}}^{k}<N-1 \\
N-1 & \text { otherwise }\end{cases} \\
& i_{\left(\Gamma_{1} \oplus \Gamma_{2}\right)_{x}}^{k}= \begin{cases}i_{\Gamma_{1_{x}}}^{k}+i_{\Gamma_{2_{x}}}^{k} & \text { if } 0<i_{\Gamma_{1_{x}}}^{k}+i_{\Gamma_{2_{x}}}^{k} \leq N-1 \\
0 & \text { otherwise }\end{cases} \\
& f_{\left(\Gamma_{1} \oplus \Gamma_{2}\right)_{x}}^{k}= \begin{cases}f_{\Gamma_{1_{x}}}^{k}+f_{\Gamma_{2_{x}}}^{k} & \text { if } 0<f_{\Gamma_{1_{x}}}^{k}+f_{\Gamma_{2_{x}}}^{k} \leq N-1 \\
0 & \text { otherwise }\end{cases}
\end{aligned}
$$

for all $u_{k} \in \mathcal{U}$.

Definition 3.8. A subtraction of NN-SS $\left(\Gamma_{1}, \mathcal{E}, N\right)$ and $\left(\Gamma_{2}, \mathcal{E}, N\right)$ is defined by $\left(\Gamma_{1}, \mathcal{E}, N\right) \ominus\left(\Gamma_{2}, \mathcal{E}, N\right)=\left(\Gamma_{1} \ominus \Gamma_{2}, \mathcal{E}, N\right)$, where $\left(\Gamma_{1} \ominus \Gamma_{2}\right)(x)=\left\{\left(u_{k}:\left\langle t_{\left(\Gamma_{1} \ominus \Gamma_{2}\right)_{x}}^{k}, i_{\left(\Gamma_{1} \ominus \Gamma_{2}\right)_{x}}^{k}, f_{\left(\Gamma_{1} \ominus \Gamma_{2}\right)_{x}}^{k}\right)\right): u_{k} \in \mathcal{U}\right\}$ for every $x \in \mathcal{E}$ such that

$$
\begin{aligned}
t_{\left(\Gamma_{1} \ominus \Gamma_{2}\right)_{x}}^{k} & = \begin{cases}t_{\Gamma_{1_{x}}}^{k}-t_{\Gamma_{2 x}}^{k} & \text { if } t_{\Gamma_{1_{x}}}^{k}>t_{\Gamma_{2 x}}^{k} \\
0 & \text { otherwise }\end{cases} \\
i_{\left(\Gamma_{1} \ominus \Gamma_{2}\right)_{x}}^{k} & = \begin{cases}i_{\Gamma_{1_{x}}}^{k}-i_{\Gamma_{2_{x}}}^{k} & \text { if } i_{\Gamma_{1_{x}}}^{k}>i_{\Gamma_{2_{x}}}^{k} \\
N-1 & \text { otherwise }\end{cases} \\
f_{\left(\Gamma_{1} \ominus \Gamma_{2}\right)_{x}}^{k} & = \begin{cases}f_{\Gamma_{1_{x}}}^{k}-f_{\Gamma_{2_{x}}}^{k} & \text { if } f_{\Gamma_{1_{x}}}^{k}>f_{\Gamma_{2_{x}}}^{k} \\
N-1 & \text { otherwise }\end{cases}
\end{aligned}
$$

for all $u_{k} \in \mathcal{U}$. 
Example 3.9. Let's consider the N8-SSs $\left(\Gamma_{3}, \mathcal{E}, 8\right)$ given below and $\left(\Gamma_{2}, \mathcal{E}, 8\right)$ of Example 2.11. Then their addition and subtraction are given in $\mathbf{M}_{\boldsymbol{\Gamma}_{3} \oplus \boldsymbol{\Gamma}_{2}}$ and $\mathbf{M}_{\boldsymbol{\Gamma}_{3} \ominus \boldsymbol{\Gamma}_{2}}$, respectively,

$$
\begin{aligned}
\mathbf{M}_{\boldsymbol{\Gamma}_{3}}= & \left(\begin{array}{llll}
\langle 7,7,4\rangle & \langle 1,4,2\rangle & \langle 7,3,2\rangle & \langle 7,1,3\rangle \\
\langle 1,5,5\rangle & \langle 2,1,4\rangle & \langle 2,1,5\rangle & \langle 2,2,3\rangle \\
\langle 3,3,3\rangle & \langle 7,3,1\rangle & \langle 7,2,1\rangle & \langle 1,5,1\rangle
\end{array}\right), \\
\mathbf{M}_{\boldsymbol{\Gamma}_{\mathbf{3}} \oplus \boldsymbol{\Gamma}_{\mathbf{2}}} & =\left(\begin{array}{llll}
\langle 7,0,0\rangle & \langle 5,7,5\rangle & \langle 7,3,5\rangle & \langle 7,3,7\rangle \\
\langle 7,7,0\rangle & \langle 5,3,4\rangle & \langle 6,3,6\rangle & \langle 7,5,4\rangle \\
\langle 7,3,5\rangle & \langle 7,7,3\rangle & \langle 7,5,3\rangle & \langle 6,7,4\rangle
\end{array}\right), \\
\mathbf{M}_{\boldsymbol{\Gamma}_{3} \oplus \boldsymbol{\Gamma}_{\mathbf{2}}} & =\left(\begin{array}{llll}
\langle 1,6,7\rangle & \langle 0,1,7\rangle & \langle 1,3,7\rangle & \langle 1,7,7\rangle \\
\langle 0,3,2\rangle & \langle 0,7,4\rangle & \langle 0,7,4\rangle & \langle 0,7,2\rangle \\
\langle 0,3,1\rangle & \langle 2,7,7\rangle & \langle 1,7,7\rangle & \langle 0,3,7\rangle
\end{array}\right) .
\end{aligned}
$$

Proposition 3.10. Let $(\Gamma, \mathcal{E}, N),\left(\Gamma_{1}, \mathcal{E}, N\right),\left(\Gamma_{2}, \mathcal{E}, N\right),\left(\Gamma_{3}, \mathcal{E}, N\right) \in N N-S S(\mathcal{U})$. Then, i. $\left(\Gamma_{1}, \mathcal{E}, N\right) \oplus\left(\Gamma_{2}, \mathcal{E}, N\right)=\left(\Gamma_{2}, \mathcal{E}, N\right) \oplus\left(\Gamma_{1}, \mathcal{E}, N\right)$.

ii. $\left[\left(\Gamma_{1}, \mathcal{E}, N\right) \oplus\left(\Gamma_{2}, \mathcal{E}, N\right)\right] \oplus\left(\Gamma_{3}, \mathcal{E}, N\right)=\left(\Gamma_{1}, \mathcal{E}, N\right) \oplus\left[\left(\Gamma_{2}, \mathcal{E}, N\right) \oplus\left(\Gamma_{3}, \mathcal{E}, N\right)\right]$.

iii. $(\Gamma, \mathcal{E}, N) \oplus\left(\Gamma_{\mathcal{U}}, \mathcal{E}, N\right)=\left(\Gamma_{\mathcal{U}}, \mathcal{E}, N\right)$.

iv. $(\Gamma, \mathcal{E}, N) \oplus\left(\Gamma_{\varnothing}, \mathcal{E}, N\right)=(\Gamma, \mathcal{E}, N)$.

v. $(\Gamma, \mathcal{E}, N) \ominus\left(\Gamma_{\varnothing}, \mathcal{E}, N\right)=(\Gamma, \mathcal{E}, N)$.

vi. If $\left(\Gamma_{1}, \mathcal{E}, N\right) \sqsubseteq\left(\Gamma_{2}, \mathcal{E}, N\right)$, then $\left(\Gamma_{1}, \mathcal{E}, N\right) \ominus\left(\Gamma_{2}, \mathcal{E}, N\right)=\left(\Gamma_{\varnothing}, \mathcal{E}, N\right)$.

Proof. The proofs of $i .-v$. follow from Definition 3.7 and 3.8.

$v i$. Let $x \in \mathcal{E}$. From our assumption, $t_{\Gamma_{1_{x}}}^{k} \leq t_{\Gamma_{2 x}}^{k}, i_{\Gamma_{1_{x}}}^{k} \geq i_{\Gamma_{2_{x}}}^{k}$ and $f_{\Gamma_{1_{x}}}^{k} \geq f_{\Gamma_{2_{x}}}^{k}$ for all $u_{k} \in \mathcal{U}$. It implies that $t_{\left(\Gamma_{1} \ominus \Gamma_{2}\right)_{x}}^{k}=0$, $i_{\left(\Gamma_{1} \ominus \Gamma_{2}\right)_{x}}^{k}=N-1$ and $f_{\left(\Gamma_{1} \ominus \Gamma_{2}\right)_{x}}^{k}=N-1$ for all $u_{k} \in \mathcal{U}$, and hence proof of $v i$..

Example 3.11. The results $i$. and ii. of Proposition 3.10 are not true when we replace $\oplus$ by $\ominus$. Consider the N8-NSs $\left(\Gamma_{1}, \mathcal{E}, N\right),\left(\Gamma_{2}, \mathcal{E}, N\right)$ of Example 2.11 and $\left(\Gamma_{3}, \mathcal{E}, N\right)$ of Example 3.9. It can be easily verified that

- if $\Lambda_{11}^{\Gamma_{1} \ominus \Gamma_{2}}=\langle 0,1,2\rangle \neq\langle 1,7,7\rangle=\Lambda_{11}^{\Gamma_{2} \ominus \Gamma_{1}}$, then $\left(\Gamma_{1}, \mathcal{E}, N\right) \ominus\left(\Gamma_{2}, \mathcal{E}, N\right) \neq\left(\Gamma_{2}, \mathcal{E}, N\right) \ominus\left(\Gamma_{1}, \mathcal{E}, N\right)$.

- if $\Lambda_{11}^{\left(\Gamma_{1} \ominus \Gamma_{2}\right) \ominus \Gamma_{3}}=\langle 0,7,7\rangle \neq\langle 5,7,6\rangle=\Lambda_{11}^{\Gamma_{1} \ominus\left(\Gamma_{2} \ominus \Gamma_{3}\right)}$, then $\left[\left(\Gamma_{1}, \mathcal{E}, N\right) \ominus\left(\Gamma_{2}, \mathcal{E}, N\right)\right] \ominus\left(\Gamma_{3}, \mathcal{E}, N\right) \neq\left(\Gamma_{1}, \mathcal{E}, N\right) \ominus\left[\left(\Gamma_{2}, \mathcal{E}, N\right) \ominus\right.$ $\left.\left(\Gamma_{3}, \mathcal{E}, N\right)\right]$.

Unless otherwise stated in the remainder of the study, the following information will not be repeated for NN-SSs: Let $\left(\Gamma_{h}, \mathcal{E}, N^{h}\right)$ be the N $N^{h}$-SS for $h \geq 1$. Also, it is assumed $\mathcal{K}^{h}=\left\langle T^{h}, I^{h}, F^{h}\right\rangle$ such that $T^{h}=\left\{0,1,2, \ldots, N^{h}-1\right\}=I^{h}=F^{h}$.

Definition 3.12. A union of NN-SS $\left(\Gamma_{1}, \mathcal{E}, N^{1}\right)$ and $\left(\Gamma_{2}, \mathcal{E}, N^{2}\right)$ is defined by

$$
\left(\Gamma_{1}, \mathcal{E}, N^{1}\right) \sqcup\left(\Gamma_{2}, \mathcal{E}, N^{2}\right)=\left(\Gamma_{1} \sqcup \Gamma_{2}, \mathcal{E}, \max \left\{N^{1}, N^{2}\right\}\right),
$$

where

$$
\left(\Gamma_{1} \sqcup \Gamma_{2}\right)(x)=\left\{\left(u_{k}:\left\langle t_{\left(\Gamma_{1} \sqcup \Gamma_{2}\right)_{x}}^{k}, i_{\left(\Gamma_{1} \sqcup \Gamma_{2}\right)_{x}}^{k}, f_{\left(\Gamma_{1} \sqcup \Gamma_{2}\right)_{x}}^{k}\right\rangle\right): u_{k} \in \mathcal{U}\right\}
$$

for every $x \in \mathcal{E}$ such that $t_{\left(\Gamma_{1} \sqcup \Gamma_{2}\right)_{x}}^{k}=\max \left\{t_{\left(\Gamma_{1}\right)_{x}}^{k}, t_{\left(\Gamma_{2}\right)_{x}}^{k}\right\}, i_{\left(\Gamma_{1} \sqcup \Gamma_{2}\right)_{x}}^{k}=\min \left\{i_{\left(\Gamma_{1}\right)_{x}}^{k}, i_{\left(\Gamma_{2}\right)_{x}}^{k}\right\}$ and $f_{\left(\Gamma_{1} \sqcup \Gamma_{2}\right)_{x}}^{k}=\min \left\{f_{\left(\Gamma_{1}\right)_{x}}^{k}, f_{\left(\Gamma_{2}\right)_{x}}^{k}\right\}$ for all $u_{k} \in \mathcal{U}$.

Definition 3.13. An intersection of NN-SS $\left(\Gamma_{1}, \mathcal{E}, N^{1}\right)$ and $\left(\Gamma_{2}, \mathcal{E}, N^{2}\right)$ is defined by

$$
\left(\Gamma_{1}, \mathcal{E}, N^{1}\right) \sqcap\left(\Gamma_{2}, \mathcal{E}, N^{2}\right)=\left(\Gamma_{1} \sqcap \Gamma_{2}, \mathcal{E}, \max \left\{N^{1}, N^{2}\right\}\right),
$$

where

$$
\left(\Gamma_{1} \sqcap \Gamma_{2}\right)(x)=\left\{\left(u_{k}:\left\langle t_{\left(\Gamma_{1} \sqcap \Gamma_{2}\right)_{x}}^{k}, i_{\left(\Gamma_{1} \sqcap \Gamma_{2}\right)_{x}}^{k}, f_{\left(\Gamma_{1} \sqcap \Gamma_{2}\right)_{x}}^{k}\right\rangle\right): u_{k} \in \mathcal{U}\right\}
$$

for every $x \in \mathcal{E}$ such that $t_{\left(\Gamma_{1} \sqcap \Gamma_{2}\right)_{x}}^{k}=\min \left\{t_{\left(\Gamma_{1}\right)_{x}}^{k}, t_{\left(\Gamma_{2}\right)_{x}}^{k}\right\}, i_{\left(\Gamma_{1} \sqcap \Gamma_{2}\right)_{x}}^{k}=\max \left\{i_{\left(\Gamma_{1}\right)_{x}}^{k}, i_{\left(\Gamma_{2}\right)_{x}}^{k}\right\}$ and $f_{\left(\Gamma_{1} \sqcap \Gamma_{2}\right)_{x}}^{k}=\max \left\{f_{\left(\Gamma_{1}\right)_{x}}^{k}, f_{\left(\Gamma_{2}\right)_{x}}^{k}\right\}$ for all $u_{k} \in \mathcal{U}$. 
Example 3.14. Let's consider the $\left(\Gamma_{4}, \mathcal{E}, 7\right)$ given below and $\left(\Gamma_{3}, \mathcal{E}, 8\right)$ of Example 3.9. Then their union and intersection are given in $\mathbf{M}_{\left(\Gamma_{3} \sqcup \Gamma_{4}\right)}$ and $\mathbf{M}_{\left(\Gamma_{3} \sqcap \Gamma_{4}\right)}$, respectively,

$$
\begin{aligned}
\mathbf{M}_{\Gamma_{4}}= & \left(\begin{array}{llll}
\langle 5,5,2\rangle & \langle 4,3,5\rangle & \langle 3,3,4\rangle & \langle 6,1,4\rangle \\
\langle 3,4,6\rangle & \langle 5,2,3\rangle & \langle 5,2,6\rangle & \langle 5,3,6\rangle \\
\langle 4,5,1\rangle & \langle 6,1,2\rangle & \langle 1,2,5\rangle & \langle 3,5,5\rangle
\end{array}\right), \\
\mathbf{M}_{\left(\Gamma_{3} \sqcup \Gamma_{4}\right)} & =\left(\begin{array}{llll}
\langle 7,2,2\rangle & \langle 4,3,2\rangle & \langle 7,3,2\rangle & \langle 7,1,3\rangle \\
\langle 3,4,5\rangle & \langle 5,1,3\rangle & \langle 5,1,5\rangle & \langle 5,2,3\rangle \\
\langle 4,3,1\rangle & \langle 7,1,1\rangle & \langle 7,2,1\rangle & \langle 3,5,1\rangle
\end{array}\right), \\
\mathbf{M}_{\left(\Gamma_{3} \sqcap \Gamma_{4}\right)} & =\left(\begin{array}{llll}
\langle 5,7,4\rangle & \langle 1,4,5\rangle & \langle 3,3,4\rangle & \langle 6,1,4\rangle \\
\langle 1,5,6\rangle & \langle 2,2,4\rangle & \langle 2,2,6\rangle & \langle 2,3,6\rangle \\
\langle 3,5,3\rangle & \langle 6,3,2\rangle & \langle 1,2,5\rangle & \langle 1,5,5\rangle
\end{array}\right) .
\end{aligned}
$$

Proposition 3.15. Let $\left(\Gamma_{1}, \mathcal{E}, N^{1}\right),\left(\Gamma_{2}, \mathcal{E}, N^{2}\right),\left(\Gamma_{3}, \mathcal{E}, N^{3}\right) \in N N-S S(\mathcal{U})$. Then,

i. $\left(\Gamma_{1}, \mathcal{E}, N^{1}\right) \star\left(\Gamma_{1}, \mathcal{E}, N^{1}\right)=\left(\Gamma_{1}, \mathcal{E}, N^{1}\right)$ for each $\star \in\{\sqcup, \sqcap\}$.

ii. $\left(\Gamma_{1}, \mathcal{E}, N^{1}\right) \star\left(\Gamma_{2}, \mathcal{E}, N^{2}\right)=\left(\Gamma_{2}, \mathcal{E}, N^{2}\right) \star\left(\Gamma_{1}, \mathcal{E}, N^{1}\right)$ for each $\star \in\{\sqcup, \Pi\}$.

iii. $\left(\Gamma_{1}, \mathcal{E}, N^{1}\right) \star\left[\left(\Gamma_{2}, \mathcal{E}, N^{2}\right) \star\left(\Gamma_{3}, \mathcal{E}, N^{3}\right)\right]=\left[\left(\Gamma_{1}, \mathcal{E}, N^{1}\right) \star\left(\Gamma_{2}, \mathcal{E}, N^{2}\right)\right] \star\left(\Gamma_{3}, \mathcal{E}, N^{3}\right)$ for each $\star \in\{\sqcup, \Pi\}$.

iv. If $\left(\Gamma_{1}, \mathcal{E}, N^{1}\right) \sqsubseteq\left(\Gamma_{2}, \mathcal{E}, N^{2}\right)$, then $\left(\Gamma_{1}, \mathcal{E}, N^{1}\right) \sqcup\left(\Gamma_{2}, \mathcal{E}, N^{2}\right)=\left(\Gamma_{2}, \mathcal{E}, N^{2}\right)$.

v. If $\left(\Gamma_{1}, \mathcal{E}, N^{1}\right) \sqsubseteq\left(\Gamma_{2}, \mathcal{E}, N^{2}\right)$, then $\left(\Gamma_{1}, \mathcal{E}, N^{1}\right) \sqcap\left(\Gamma_{2}, \mathcal{E}, N^{2}\right)=\left(\Gamma_{1}, \mathcal{E}, N^{1}\right)$.

vi. $\left(\Gamma_{1}, \mathcal{E}, N^{1}\right) \star\left[\left(\Gamma_{2}, \mathcal{E}, N^{2}\right) \star\left(\Gamma_{3}, \mathcal{E}, N^{3}\right)\right]=\left[\left(\Gamma_{1}, \mathcal{E}, N^{1}\right) \star\left(\Gamma_{2}, \mathcal{E}, N^{2}\right)\right] *\left[\left(\Gamma_{1}, \mathcal{E}, N^{1}\right) \star\left(\Gamma_{3}, \mathcal{E}, N^{3}\right)\right]$ for each $\star \in\{\sqcup, \sqcap\}$. vii. $\left[\left(\Gamma_{1}, \mathcal{E}, N^{1}\right) \star\left(\Gamma_{2}, \mathcal{E}, N^{2}\right)\right]^{c}=\left(\Gamma_{1}, \mathcal{E}, N^{1}\right)^{c} *\left(\Gamma_{2}, \mathcal{E}, N^{2}\right)^{c}$ for each $\star \in\{\sqcup, \Pi\}$ such that $\star \neq *$.

Proof. The proof is clear from Definitions 2.9, 3.1, 3.12 and 3.13.

Definition 3.16. An Or-product of NN-SS $\left(\Gamma_{1}, \mathcal{E}, N^{1}\right)$ and $\left(\Gamma_{2}, \mathcal{E}, N^{2}\right)$ is defined by $\left(\Gamma_{1}, \mathcal{E}, N^{1}\right) \vee\left(\Gamma_{2}, \mathcal{E}, N^{2}\right)=\left(\Gamma_{1} \vee\right.$ $\left.\Gamma_{2}, \mathcal{E} \times \mathcal{E}, \max \left\{N^{1}, N^{2}\right\}\right)$, where

$$
\left(\Gamma_{1} \vee \Gamma_{2}\right)\left(x_{r}, x_{s}\right)=\left\{\left(u_{k}:<t_{\left(\Gamma_{1} \vee \Gamma_{2}\right)\left(x_{r}, x_{s}\right)}^{k}, i_{\left(\Gamma_{1} \vee \Gamma_{2}\right)\left(x_{r}, x_{s}\right)}^{k}, f_{\left(\Gamma_{1} \vee \Gamma_{2}\right)\left(x_{r}, x_{s}\right)}^{k}>\right): u_{k} \in \mathcal{U}\right\}
$$

for every $\left(x_{r}, x_{s}\right) \in \mathcal{E} \times \mathcal{E}$ such that $t_{\left(\Gamma_{1} \vee \Gamma_{2}\right)\left(x_{r}, x_{s}\right)}^{k}=\max \left\{\left[t_{\left(\Gamma_{1}\right)_{x}}^{1}\right]^{k},\left[t_{\left(\Gamma_{2}\right)_{x_{s}}}^{2}\right]^{k}\right\}, i_{\left(\Gamma_{1} \vee \Gamma_{2}\right)\left(x_{r}, x_{s}\right)}^{k}=\min \left\{\left[i_{\left(\Gamma_{1}\right)_{x r}}^{1}\right]^{k},\left[i_{\left(\Gamma_{2}\right)_{x_{s}}}^{2}\right]^{k}\right\}$ and $f_{\left(\Gamma_{1} \vee \Gamma_{2}\right)\left(x_{r}, x_{s}\right)}^{k}=\min \left\{\left[f_{\left(\Gamma_{1}\right)_{x_{r}}}^{1}\right]^{k},\left[f_{\left(\Gamma_{2}\right)_{x_{s}}}^{2}\right]^{k}\right\}$ for all $u_{k} \in \mathcal{U}$. Also,

$i$. The Or-Top-Not-product of $\left(\Gamma_{1}, \mathcal{E}, N^{1}\right)$ and $\left(\Gamma_{2}, \mathcal{E}, N^{2}\right)$ is defined as

$$
\left(\Gamma_{1}, \mathcal{E}, N^{1}\right) \underline{\vee_{t w}}\left(\Gamma_{2}, \mathcal{E}, N^{2}\right)=\left(\Gamma_{1}, \mathcal{E}, N^{1}\right) \vee\left(\Gamma_{2}, \mathcal{E}, N^{2}\right)^{t w} .
$$

ii. The Or-Bottom-Not-product of $\left(\Gamma_{1}, \mathcal{E}, N^{1}\right)$ and $\left(\Gamma_{2}, \mathcal{E}, N^{2}\right)$ is defined as

$$
\left(\Gamma_{1}, \mathcal{E}, N^{1}\right) \underline{\bigvee_{b w}}\left(\Gamma_{2}, \mathcal{E}, N^{2}\right)=\left(\Gamma_{1}, \mathcal{E}, N^{1}\right) \vee\left(\Gamma_{2}, \mathcal{E}, N^{2}\right)^{b w} .
$$

iii. The Or-Not-product of $\left(\Gamma_{1}, \mathcal{E}, N^{1}\right)$ and $\left(\Gamma_{2}, \mathcal{E}, N^{2}\right)$ is defined as

$$
\left(\Gamma_{1}, \mathcal{E}, N^{1}\right) \underline{\vee}\left(\Gamma_{2}, \mathcal{E}, N^{2}\right)=\left(\Gamma_{1}, \mathcal{E}, N^{1}\right) \vee\left(\Gamma_{2}, \mathcal{E}, N^{2}\right)^{c} .
$$

Example 3.17. Let $\mathcal{U}=\left\{u_{1}, u_{2}, u_{3}\right\}$ be a universal set and $\mathcal{E}=\left\{x_{1}, x_{2}\right\}$ be a parameter set. We consider the N5-SS $\left(\Gamma_{\star}, \mathcal{E}, 5\right)$ in $\mathbf{M}_{\Gamma_{\star}}$ and the N6-SS $\left(\Gamma_{*}, \mathcal{E}, 5\right)$ in $\mathbf{M}_{\Gamma_{*}}$.

$$
\begin{gathered}
\mathbf{M}_{\Gamma_{*}}=\left(\begin{array}{cc}
\langle 3,1,4\rangle & \langle 1,0,2\rangle \\
\langle 4,2,3\rangle & \langle 4,2,3\rangle \\
\langle 2,3,1\rangle & \langle 3,2,4\rangle
\end{array}\right) \mathbf{M}_{\Gamma_{*}}=\left(\begin{array}{cc}
\langle 5,0,3\rangle & \langle 1,0,5\rangle \\
\langle 3,4,2\rangle & \langle 5,4,5\rangle \\
\langle 1,3,5\rangle & \langle 4,3,2\rangle
\end{array}\right) \\
\mathbf{M}_{\Gamma_{*}^{\text {tw }}}=\left(\begin{array}{cc}
\langle 0,5,0\rangle & \langle 5,5,0\rangle \\
\langle 5,0,0\rangle & \langle 0,0,0\rangle \\
\langle 5,0,0\rangle & \langle 5,0,0\rangle
\end{array}\right) \mathbf{M}_{\Gamma_{*}^{b w}}=\left(\begin{array}{cc}
\langle 0,5,5\rangle & \langle 0,5,0\rangle \\
\langle 0,5,5\rangle & \langle 0,5,0\rangle \\
\langle 0,5,0\rangle & \langle 0,5,5\rangle
\end{array}\right) \\
\mathbf{M}_{\Gamma_{*}^{c}}=\left(\begin{array}{cc}
\langle 3,5,5\rangle & \langle 5,5,1\rangle \\
\langle 2,1,3\rangle & \langle 5,1,5\rangle \\
\langle 5,2,1\rangle & \langle 2,2,4\rangle
\end{array}\right) .
\end{gathered}
$$


Then their the Or-product, Or-Top-Not-product, Or-Bottom-Not-product and Or-Not-product are given in $\left(\Gamma_{*} \vee \Gamma_{*}, \mathcal{E} \times\right.$ $\mathcal{E}, 6),\left(\Gamma_{*} \vee_{t w} \Gamma_{*}, \mathcal{E} \times \mathcal{E}, 6\right),\left(\Gamma_{*} \underline{\vee b w}_{b *}, \mathcal{E} \times \mathcal{E}, 6\right)$ and $\left(\Gamma_{*} \underline{\vee} \Gamma_{*}, \mathcal{E} \times \mathcal{E}, 6\right)$, respectively,

$$
\begin{gathered}
\left(\Gamma_{*} \vee \Gamma_{*}, \mathcal{E} \times \mathcal{E}, 6\right)=\left\{\begin{array}{c}
\left(\left(x_{1}, x_{1}\right),\left(u_{1},\langle 5,0,3\rangle\right),\left(u_{2},\langle 4,2,2\rangle\right),\left(u_{3},\langle 2,3,1\rangle\right)\right), \\
\left(\left(x_{1}, x_{2}\right),\left(u_{1},\langle 3,0,4\rangle\right),\left(u_{2},\langle 5,2,3\rangle\right),\left(u_{3},\langle 4,3,1\rangle\right)\right), \\
\left(\left(x_{2}, x_{1}\right),\left(u_{1},\langle 5,0,2\rangle\right),\left(u_{2},\langle 4,2,2\rangle\right),\left(u_{3},\langle 3,2,4\rangle\right)\right), \\
\left(\left(x_{2}, x_{2}\right),\left(u_{1},\langle 1,0,2\rangle\right),\left(u_{2},\langle 5,2,3\rangle\right),\left(u_{3},\langle 4,2,2\rangle\right)\right)
\end{array}\right\}, \\
\left(\Gamma_{*} \underline{\vee}_{t w} \Gamma_{*}, \mathcal{E} \times \mathcal{E}, 6\right)=\left\{\begin{array}{c}
\left(\left(x_{1}, x_{1}\right),\left(u_{1},\langle 3,1,0\rangle\right),\left(u_{2},\langle 5,0,0\rangle\right),\left(u_{3},\langle 5,0,0\rangle\right)\right), \\
\left(\left(x_{1}, x_{2}\right),\left(u_{1},\langle 5,1,0\rangle\right),\left(u_{2},\langle 4,0,0\rangle\right),\left(u_{3},\langle 5,0,0\rangle\right)\right), \\
\left(\left(x_{2}, x_{1}\right),\left(u_{1},\langle 1,0,0\rangle\right),\left(u_{2},\langle 5,0,0\rangle\right),\left(u_{3},\langle 3,2,4\rangle\right)\right), \\
\left(\left(x_{2}, x_{2}\right),\left(u_{1},\langle 5,0,0\rangle\right),\left(u_{2},\langle 4,0,0\rangle\right),\left(u_{3},\langle 5,0,0\rangle\right)\right)
\end{array}\right\}, \\
\left(\Gamma_{*} \underline{\vee}_{b w} \Gamma_{*}, \mathcal{E} \times \mathcal{E}, 6\right)=\left\{\begin{array}{c}
\left(\left(x_{1}, x_{1}\right),\left(u_{1},\langle 3,1,4\rangle\right),\left(u_{2},\langle 4,2,3\rangle\right),\left(u_{3},\langle 2,3,0\rangle\right)\right), \\
\left(\left(x_{1}, x_{2}\right),\left(u_{1},\langle 3,1,0\rangle\right),\left(u_{2},\langle 4,2,0\rangle\right),\left(u_{3},\langle 2,3,1\rangle\right)\right), \\
\left(\left(x_{2}, x_{1}\right),\left(u_{1},\langle 1,0,2\rangle\right),\left(u_{2},\langle 4,2,3\rangle\right),\left(u_{3},\langle 3,2,4\rangle\right)\right), \\
\left(\left(x_{2}, x_{2}\right),\left(u_{1},\langle 1,0,0\rangle\right),\left(u_{2},\langle 4,2,0\rangle\right),\left(u_{3},\langle 3,2,2\rangle\right)\right)
\end{array}\right\}, \\
\left(\Gamma_{*} \underline{\vee} \Gamma_{*}, \mathcal{E} \times \mathcal{E}, 6\right)=\left\{\begin{array}{c}
\left(\left(x_{1}, x_{1}\right),\left(u_{1},\langle 3,1,4\rangle\right),\left(u_{2},\langle 4,1,3\rangle\right),\left(u_{3},\langle 5,2,1\rangle\right)\right), \\
\left(\left(x_{1}, x_{2}\right),\left(u_{1},\langle 5,1,1\rangle\right),\left(u_{2},\langle 5,1,3\rangle\right),\left(u_{3},\langle 2,2,1\rangle\right)\right), \\
\left(\left(x_{2}, x_{1}\right),\left(u_{1},\langle 3,0,2\rangle\right),\left(u_{2},\langle 4,1,3\rangle\right),\left(u_{3},\langle 5,2,1\rangle\right)\right), \\
\left(\left(x_{2}, x_{2}\right),\left(u_{1},\langle 5,0,1\rangle\right),\left(u_{2},\langle 5,1,3\rangle\right),\left(u_{3},\langle 3,2,4\rangle\right)\right)
\end{array}\right\},
\end{gathered}
$$

Definition 3.18. An And-product of NN-SS $\left(\Gamma_{1}, \mathcal{E}, N^{1}\right)$ and $\left(\Gamma_{2}, \mathcal{E}, N^{2}\right)$ is defined by $\left(\Gamma_{1}, \mathcal{E}, N^{1}\right) \wedge\left(\Gamma_{2}, \mathcal{E}, N^{2}\right)=\left(\Gamma_{1} \wedge\right.$ $\left.\Gamma_{2}, \mathcal{E} \times \mathcal{E}, \max \left\{N^{1}, N^{2}\right\}\right)$, where

$$
\left(\Gamma_{1} \wedge \Gamma_{2}\right)\left(x_{r}, x_{s}\right)=\left\{\left(u_{k}:<t_{\left(\Gamma_{1} \wedge \Gamma_{2}\right)\left(x_{r}, x_{s}\right)}^{k}, i_{\left(\Gamma_{1} \wedge \Gamma_{2}\right)\left(x_{r}, x_{s}\right)}^{k}, f_{\left(\Gamma_{1} \wedge \Gamma_{2}\right)\left(x_{r}, x_{s}\right)}^{k}>\right): u_{k} \in \mathcal{U}\right\}
$$

for every $\left(x_{r}, x_{s}\right) \in \mathcal{E} \times \mathcal{E}$ such that $t_{\left(\Gamma_{1} \wedge \Gamma_{2}\right)\left(x_{r}, x_{s}\right)}^{k}=\min \left\{\left[t_{\left(\Gamma_{1}\right) x_{r}}^{1}\right]^{k},\left[t_{\left(\Gamma_{2}\right) x_{s}}^{2}\right]^{k}\right\}, i_{\left(\Gamma_{1} \wedge \Gamma_{2}\right)\left(x_{r}, x_{s}\right)}^{k}=\max \left\{\left[i_{\left(\Gamma_{1}\right) x_{r}}^{1}\right]^{k},\left[i_{\left(\Gamma_{2}\right) x_{s}}^{2}\right]^{k}\right\}$ and $f_{\left(\Gamma_{1} \wedge \Gamma_{2}\right)\left(x_{r}, x_{s}\right)}^{k}=\max \left\{\left[f_{\left(\Gamma_{1}\right)_{x_{r}}}^{1}\right]^{k},\left[f_{\left(\Gamma_{2}\right)_{x_{s}}}^{2}\right]^{k}\right\}$ for all $u_{k} \in \mathcal{U}$. Also,

$i$. The And-Top-Not-product of $\left(\Gamma_{1}, \mathcal{E}, N^{1}\right)$ and $\left(\Gamma_{2}, \mathcal{E}, N^{2}\right)$ is defined as

$$
\left(\Gamma_{1}, \mathcal{E}, N^{1}\right) \overline{\Lambda_{t w}}\left(\Gamma_{2}, \mathcal{E}, N^{2}\right)=\left(\Gamma_{1}, \mathcal{E}, N^{1}\right) \wedge\left(\Gamma_{2}, \mathcal{E}, N^{2}\right)^{t w} .
$$

ii. The And-Bottom-Not-product of $\left(\Gamma_{1}, \mathcal{E}, N^{1}\right)$ and $\left(\Gamma_{2}, \mathcal{E}, N^{2}\right)$ is defined as

$$
\left(\Gamma_{1}, \mathcal{E}, N^{1}\right) \overline{\wedge_{b w}}\left(\Gamma_{2}, \mathcal{E}, N^{2}\right)=\left(\Gamma_{1}, \mathcal{E}, N^{1}\right) \wedge\left(\Gamma_{2}, \mathcal{E}, N^{2}\right)^{b w} .
$$

iii. The And-Not-product of $\left(\Gamma_{1}, \mathcal{E}, N^{1}\right)$ and $\left(\Gamma_{2}, \mathcal{E}, N^{2}\right)$ is defined as

$$
\left(\Gamma_{1}, \mathcal{E}, N^{1}\right) \bar{\wedge}\left(\Gamma_{2}, \mathcal{E}, N^{2}\right)=\left(\Gamma_{1}, \mathcal{E}, N^{1}\right) \wedge\left(\Gamma_{2}, \mathcal{E}, N^{2}\right)^{c} .
$$

Example 3.19. Consider the N5-SS $\left(\Gamma_{\star}, \mathcal{E}, 5\right)$ and the N6-SS $\left(\Gamma_{\star}, \mathcal{E}, 6\right)$ as given in Example 3.17. Then their the And-product, And-Top-Not-product, And-Bottom-Not-product and And-Not-product are given in $\left(\Gamma_{*} \wedge \Gamma_{*}, \mathcal{E} \times \mathcal{E}, 6\right)$, $\left(\Gamma_{*} \overline{\Lambda_{t w}} \Gamma_{*}, \mathcal{E} \times \mathcal{E}, 6\right),\left(\Gamma_{*} \overline{\Lambda_{b w}} \Gamma_{*}, \mathcal{E} \times \mathcal{E}, 6\right)$ and $\left(\Gamma_{*} \bar{\wedge} \Gamma_{*}, \mathcal{E} \times \mathcal{E}, 6\right)$, respectively,

$$
\begin{gathered}
\left(\Gamma_{*} \wedge \Gamma_{*}, \mathcal{E} \times \mathcal{E}, 6\right)=\left\{\begin{array}{c}
\left(\left(x_{1}, x_{1}\right),\left(u_{1},\langle 3,1,4\rangle\right),\left(u_{2},\langle 3,4,3\rangle\right),\left(u_{3},\langle 1,3,5\rangle\right)\right), \\
\left(\left(x_{1}, x_{2}\right),\left(u_{1},\langle 1,1,5\rangle\right),\left(u_{2},\langle 4,4,5\rangle\right),\left(u_{3},\langle 2,3,2\rangle\right)\right), \\
\left(\left(x_{2}, x_{1}\right),\left(u_{1},\langle 1,0,3\rangle\right),\left(u_{2},\langle 3,4,3\rangle\right),\left(u_{3},\langle 1,3,5\rangle\right)\right), \\
\left(\left(x_{2}, x_{2}\right),\left(u_{1},\langle 1,0,5\rangle\right),\left(u_{2},\langle 4,4,5\rangle\right),\left(u_{3},\langle 3,3,4\rangle\right)\right)
\end{array}\right\}, \\
\left(\Gamma_{*} \overline{\Lambda_{t w}} \Gamma_{*}, \mathcal{E} \times \mathcal{E}, 6\right)=\left\{\begin{array}{c}
\left(\left(x_{1}, x_{1}\right),\left(u_{1},\langle 0,5,4\rangle\right),\left(u_{2},\langle 4,2,3\rangle\right),\left(u_{3},\langle 2,3,1\rangle\right)\right), \\
\left(\left(x_{1}, x_{2}\right),\left(u_{1},\langle 3,5,4\rangle\right),\left(u_{2},\langle 0,2,3\rangle\right),\left(u_{3},\langle 2,3,1\rangle\right)\right) \\
\left(\left(x_{2}, x_{1}\right),\left(u_{1},\langle 0,5,2\rangle\right),\left(u_{2},\langle 4,2,3\rangle\right),\left(u_{3},\langle 3,2,4\rangle\right)\right), \\
\left(\left(x_{2}, x_{2}\right),\left(u_{1},\langle 1,5,2\rangle\right),\left(u_{2},\langle 0,2,3\rangle\right),\left(u_{3},\langle 3,2,4\rangle\right)\right)
\end{array}\right\}, \\
\left(\Gamma_{*}{\overline{\Lambda_{b w}}} \Gamma_{*}, \mathcal{E} \times \mathcal{E}, 6\right)=\left\{\begin{array}{c}
\left(\left(x_{1}, x_{1}\right),\left(u_{1},\langle 0,5,5\rangle\right),\left(u_{2},\langle 0,5,5\rangle\right),\left(u_{3},\langle 0,5,1\rangle\right)\right), \\
\left(\left(x_{1}, x_{2}\right),\left(u_{1},\langle 0,5,4\rangle\right),\left(u_{2},\langle 0,5,3\rangle\right),\left(u_{3},\langle 0,5,5\rangle\right)\right), \\
\left(\left(x_{2}, x_{1}\right),\left(u_{1},\langle 0,5,5\rangle\right),\left(u_{2},\langle 0,5,5\rangle\right),\left(u_{3},\langle 0,5,4\rangle\right)\right), \\
\left(\left(x_{2}, x_{2}\right),\left(u_{1},\langle 0,5,2\rangle\right),\left(u_{2},\langle 0,5,3\rangle\right),\left(u_{3},\langle 0,5,5\rangle\right)\right)
\end{array}\right\},
\end{gathered}
$$




$$
\left(\Gamma_{*} \bar{\wedge} \Gamma_{*}, \mathcal{E} \times \mathcal{E}, 6\right)=\left\{\begin{array}{c}
\left(\left(x_{1}, x_{1}\right),\left(u_{1},\langle 3,5,5\rangle\right),\left(u_{2},\langle 2,2,3\rangle\right),\left(u_{3},\langle 2,3,1\rangle\right)\right), \\
\left(\left(x_{1}, x_{2}\right),\left(u_{1},\langle 3,5,4\rangle\right),\left(u_{2},\langle 4,2,5\rangle\right),\left(u_{3},\langle 2,3,4\rangle\right)\right), \\
\left(\left(x_{2}, x_{1}\right),\left(u_{1},\langle 1,5,5\rangle\right),\left(u_{2},\langle 2,2,3\rangle\right),\left(u_{3},\langle 3,2,4\rangle\right)\right), \\
\left(\left(x_{2}, x_{2}\right),\left(u_{1},\langle 1,5,2\rangle\right),\left(u_{2},\langle 4,2,5\rangle\right),\left(u_{3},\langle 2,2,4\rangle\right)\right)
\end{array}\right\} .
$$

Proposition 3.20. Let $\left(\Gamma_{1}, \mathcal{E}, N^{1}\right),\left(\Gamma_{2}, \mathcal{E}, N^{2}\right) \in N N-S S(\mathcal{U})$. Then we have the following only if $N_{1}=N_{2}$. i. $\left[\left(\Gamma_{1}, \mathcal{E}, N^{1}\right) \vee\left(\Gamma_{2}, \mathcal{E}, N^{2}\right)\right]^{c}=\left(\Gamma_{1}, \mathcal{E}, N^{1}\right)^{c} \wedge\left(\Gamma_{2}, \mathcal{E}, N^{2}\right)^{c}$. ii. $\left[\left(\Gamma_{1}, \mathcal{E}, N^{1}\right) \wedge\left(\Gamma_{2}, \mathcal{E}, N^{2}\right)\right]^{c}=\left(\Gamma_{1}, \mathcal{E}, N^{1}\right)^{c} \vee\left(\Gamma_{2}, \mathcal{E}, N^{2}\right)^{c}$.

Proof. $i$. From Definitions 3.1, 3.16 and 3.18, we can write for every $\left(x_{r}, x_{s}\right) \in \mathcal{E} \times \mathcal{E}$ and for each $u_{k} \in \mathcal{U}$

$$
\begin{aligned}
& t_{\left(\Gamma_{1} \wedge \Gamma_{2}\right)^{c}\left(x_{r}, x_{s}\right)}^{k}=f_{\left(\Gamma_{1} \wedge \Gamma_{2}\right)_{\left(x r, x_{s}\right)}}^{k}=\min \left\{\left[f_{\left(\Gamma_{1}\right)_{x_{r}}}^{1}\right]^{k},\left[f_{\left(\Gamma_{2}\right)_{x_{s}}}^{2}\right]^{k}\right\} \\
& =\min \left\{\left[t_{\left(\Gamma_{1}\right)^{c} x_{r}}^{1}\right]^{k},\left[t_{\left(\Gamma_{2}\right)^{c} x_{s}}^{2}\right]^{k}\right\}=t_{\left(\Gamma_{1}^{c} \vee \Gamma_{2}^{c}\right)_{\left(x_{r}, x_{s}\right)}^{k}}^{k} \\
& i_{\left(\Gamma_{1} \wedge \Gamma_{2}\right)_{\left(x_{r}, x_{s}\right)}^{k}}^{k}=(N-1)-i_{\left(\Gamma_{1} \wedge \Gamma_{2}\right)_{\left(x_{r}, x_{s}\right)}}^{k}=(N-1)-\min \left\{\left[i_{\left(\Gamma_{1}\right)_{x r}}^{1}\right]^{k},\left[i_{\left(\Gamma_{2}\right)_{x_{s}}}^{2}\right]^{k}\right\} \\
& =\max \left\{(N-1)-\left[i_{\left(\Gamma_{1}\right)^{c} x_{r}}^{1}\right]^{k},(N-1)-\left[i_{\left(\Gamma_{2}\right)^{c} x_{s}}^{2}\right]^{k}\right\}=i_{\left(\Gamma_{1}^{c} \vee \Gamma_{2}^{c}\right)_{\left(x x_{x}, x_{s}\right)}^{k}} \\
& f_{\left(\Gamma_{1} \wedge \Gamma_{2}\right)^{c}\left(x_{r}, x_{s}\right)}^{k}=t_{\left(\Gamma_{1} \wedge \Gamma_{2}\right)\left(x_{r}, x_{s}\right)}^{k}=\min \left\{\left[t_{\left(\Gamma_{1}\right)_{x_{r}}}^{1}\right]^{k},\left[t_{\left(\Gamma_{2}\right)_{x_{s}}}^{2}\right]^{k}\right\} \\
& =\min \left\{\left[f_{\left(\Gamma_{1}\right)^{c} x_{r}}^{1}\right]^{k},\left[f_{\left(\Gamma_{2}\right)^{c} x_{s}}^{2}\right]^{k}\right\}=f_{\left.\left(\Gamma_{1}^{c} \vee \Gamma_{2}^{c}\right)_{(x p}, x_{s}\right)^{2}}^{k} .
\end{aligned}
$$

Therefore, the proof of $i$. is completed. The case $i$. can be proved similar to $i$..

\section{Similarity Measures of NN-SS}

In this section we introduce similarity measure based on the distance measures of NNSSs.

\section{Let two NN-SSs}

$$
\left(\Lambda, \mathcal{E}, N^{1}\right)=\left\{\left(x_{l},\left(u_{k},\left\langle\left[t_{\Lambda_{x}}^{1}\right]^{k},\left[i_{\Lambda_{x}}^{1}\right]^{k},\left[f_{\Lambda_{x}}^{1}\right]^{k}\right\rangle\right): u_{k} \in \mathcal{U}\right): x_{l} \in \mathcal{E}\right\}
$$

and

$$
\left(\Omega, \mathcal{E}, N^{2}\right)=\left\{\left(x_{l},\left(u_{k},\left\langle\left[t_{\Omega_{x}}^{2}\right]^{k},\left[i_{\Omega_{x}}^{2}\right]^{k},\left[f_{\Omega_{x}}^{2}\right]^{k}\right\rangle\right): u_{k} \in \mathcal{U}\right): x_{l} \in \mathcal{E}\right\}
$$

are defined over $\mathcal{U}$ for $1 \leq l \leq n$ and $1 \leq k \leq m$. Then the following Hamming and Euclidean distances are defined on $\left(\Lambda, \mathcal{E}, N^{1}\right)$ and $\left(\Omega, \mathcal{E}, N^{2}\right)$.

Hamming Distance

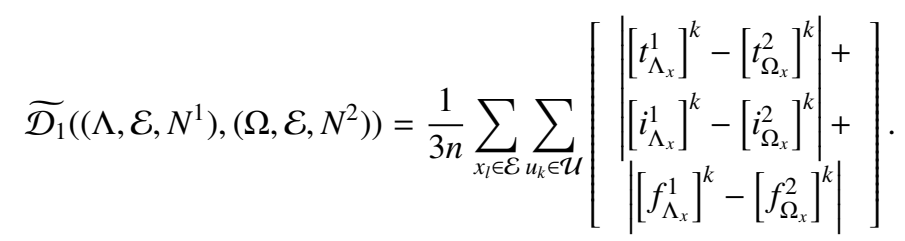

Normalised Hamming Distance

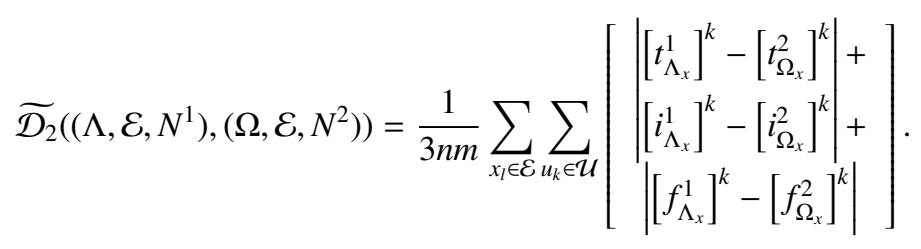


Euclidean Distance

Normalised Euclidean Distance

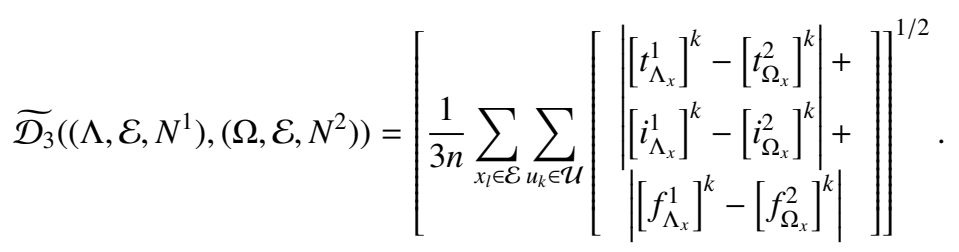

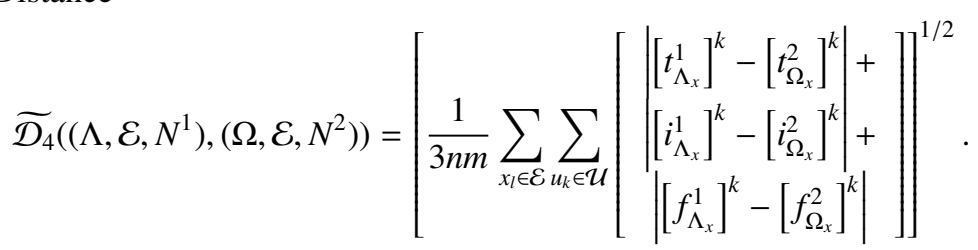

Lemma 4.1. For $N^{1} \geq N^{2}$, the following inequalities hold.

(i) $\widetilde{\mathcal{D}_{1}}\left(\left(\Lambda, \mathcal{E}, N^{1}\right),\left(\Omega, \mathcal{E}, N^{2}\right)\right) \leq m\left(N^{1}-1\right)$,

(ii) $\widetilde{\mathcal{D}_{2}}\left(\left(\Lambda, \mathcal{E}, N^{1}\right),\left(\Omega, \mathcal{E}, N^{2}\right)\right) \leq\left(N^{1}-1\right)$,

(iii) $\widetilde{\mathcal{D}_{3}}\left(\left(\Lambda, \mathcal{E}, N^{1}\right),\left(\Omega, \mathcal{E}, N^{2}\right)\right) \leq \sqrt{m\left(N^{1}-1\right)}$,

(iv) $\widetilde{\mathcal{D}_{4}}\left(\left(\Lambda, \mathcal{E}, N^{1}\right),\left(\Omega, \mathcal{E}, N^{2}\right)\right) \leq \sqrt{\left(N^{1}-1\right)}$.

Proof. Since $\left[t_{\Lambda_{x}}^{1}\right]^{k},\left[i_{\Lambda_{x}}^{1}\right]^{k},\left[f_{\Lambda_{x}}^{1}\right]^{k} \in\left[0, N^{1}-1\right]$ and $\left[t_{\Omega_{x}}^{2}\right]^{k},\left[i_{\Omega_{x}}^{2}\right]^{k},\left[f_{\Omega_{x}}^{2}\right]^{k} \in\left[0, N^{1}-1\right]$, the result holds as $\left|\left[t_{\Lambda_{x}}^{1}\right]^{k}-\left[t_{\Omega_{x}}^{2}\right]^{k}\right| \leq\left[0, N^{1}-1\right],\left|\left[i_{\Lambda_{x}}^{1}\right]^{k}-\left[i_{\Omega_{x}}^{2}\right]^{k}\right| \leq\left[0, N^{1}-1\right]$ and $\left|\left[f_{\Lambda_{x}}^{1}\right]^{k}-\left[f_{\Omega_{x}}^{2}\right]^{k}\right| \leq\left[0, N^{1}-1\right]$ for $N^{1} \geq N^{2}$.

Theorem 4.2. The above defined distances $\widetilde{\mathcal{D}_{i}}(i=1,2,3,4)$ are metrics.

Proof. We prove it for only one, as it can make similar proof for each distances.

Let $\left(\Lambda_{1}, \mathcal{E}, N^{1}\right),\left(\Lambda_{2}, \mathcal{E}, N^{2}\right),\left(\Lambda_{3}, \mathcal{E}, N^{3}\right)$ be any three NN-SSs. For $\widetilde{\mathcal{D}_{1}}$,

(i) $\widetilde{\mathcal{D}_{1}}\left(\left(\Lambda, \mathcal{E}, N^{1}\right),\left(\Omega, \mathcal{E}, N^{2}\right)\right) \geq 0$.

If $\left(\Lambda_{1}, \mathcal{E}, N^{1}\right)=\left(\Lambda_{2}, \mathcal{E}, N^{2}\right) \Rightarrow\left[\left[t_{\Lambda_{1 x}}^{1}\right]^{k}=\left[t_{\Lambda_{2 x}}^{2}\right]^{k},\left[i_{\Lambda_{1 x}}^{1}\right]^{k}=\left[i_{\Lambda_{2 x}}^{2}\right]^{k},\left[f_{\Lambda_{1 x}}^{1}\right]^{k}=\left[f_{\Lambda_{2 x}}^{2}\right]^{k} ; \forall x_{l} \in \mathcal{E}, \forall u_{k} \in \mathcal{U}\right] \Rightarrow$ $\left[\left[t_{\Lambda_{1 x}}^{1}\right]^{k}-\left[t_{\Lambda_{2 x}}^{2}\right]^{k}=0,\left[i_{\Lambda_{1 x}}^{1}\right]^{k}-\left[i_{\Lambda_{2 x}}^{2}\right]^{k}=0,\left[f_{\Lambda_{1 x}}^{1}\right]^{k}-\left[f_{\Lambda_{2 x}}^{2}\right]^{k}=0 ; \forall x_{l} \in \mathcal{E}, \forall u_{k} \in \mathcal{U}\right] \Rightarrow \widetilde{\mathcal{D}_{1}}\left(\left(\Lambda, \mathcal{E}, N^{1}\right),\left(\Omega, \mathcal{E}, N^{2}\right)\right)=0$.

Conversely, let $\widetilde{\mathcal{D}_{1}}\left(\left(\Lambda, \mathcal{E}, N^{1}\right),\left(\Omega, \mathcal{E}, N^{2}\right)\right)=0 \Rightarrow\left|\left[t_{\Lambda_{1 x}}^{1}\right]^{k}=\left[t_{\Lambda_{2 x}}^{2}\right]^{k}\right|=0,\left|\left[i_{\Lambda_{1 x}}^{1}\right]^{k}=\left[i_{\Lambda_{2 x}}^{2}\right]^{k}\right|=0,\left|\left[f_{\Lambda_{1 x}}^{1}\right]^{k}=\left[f_{\Lambda_{2 x}}^{2}\right]^{k}\right|=$ $0 \Rightarrow\left(\Lambda_{1}, \mathcal{E}, N^{1}\right)=\left(\Lambda_{2}, \mathcal{E}, N^{2}\right) \Rightarrow\left[\left[t_{\Lambda_{1 x}}^{1}\right]^{k}=\left[t_{\Lambda_{2 x}}^{2}\right]^{k},\left[i_{\Lambda_{1 x}}^{1}\right]^{k}=\left[i_{\Lambda_{2 x}}^{2}\right]^{k},\left[f_{\Lambda_{1 x}}^{1}\right]^{k}=\left[f_{\Lambda_{2 x}}^{2}\right]^{k} ; \forall x_{l} \in \mathcal{E}, \forall u_{k} \in \mathcal{U}\right] \Rightarrow$ $\left(\Lambda_{1}, \mathcal{E}, N^{1}\right)=\left(\Lambda_{2}, \mathcal{E}, N^{2}\right)$.

(ii) Clearly, $\widetilde{\mathcal{D}_{1}}\left(\left(\Lambda_{1}, \mathcal{E}, N^{1}\right),\left(\Lambda_{2}, \mathcal{E}, N^{2}\right)\right)=\widetilde{\mathcal{D}_{1}}\left(\left(\Lambda_{2}, \mathcal{E}, N^{2}\right),\left(\Lambda_{1}, \mathcal{E}, N^{1}\right)\right)$.

(iii) Finally, let's check the triangle inequality,

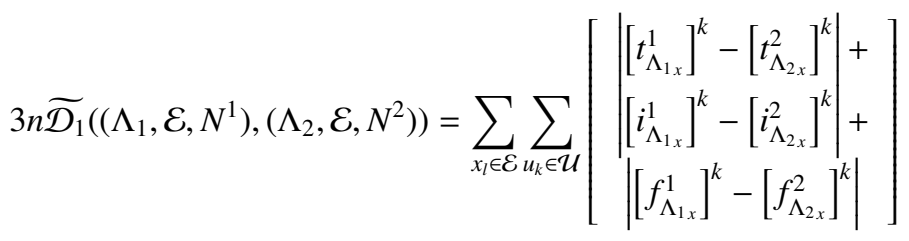

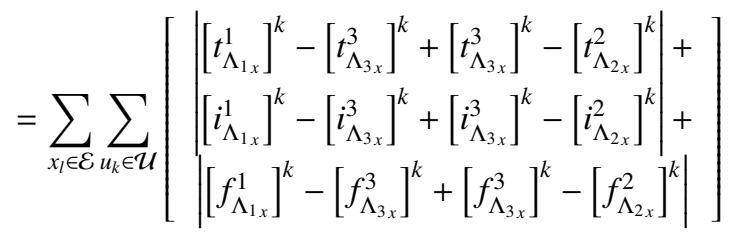




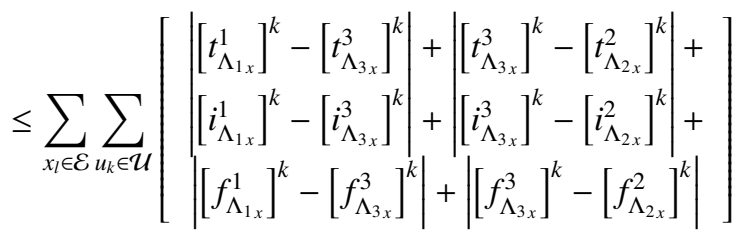

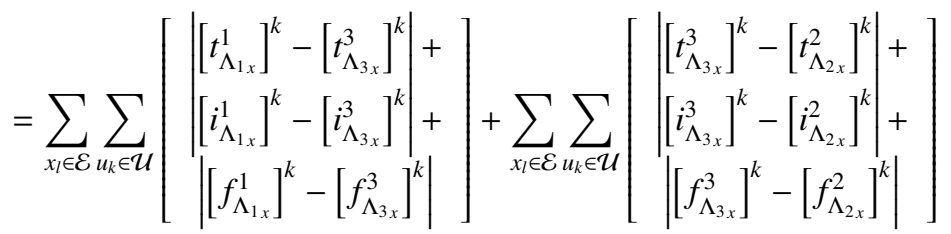

$$
\begin{aligned}
& =3 n \widetilde{\mathcal{D}_{1}}\left(\left(\Lambda_{1}, \mathcal{E}, N^{1}\right),\left(\Lambda_{3}, \mathcal{E}, N^{3}\right)\right)+3 n \widetilde{\mathcal{D}_{1}}\left(\left(\Lambda_{3}, \mathcal{E}, N^{3}\right),\left(\Lambda_{2}, \mathcal{E}, N^{2}\right)\right),
\end{aligned}
$$

i.e., $3 n \widetilde{\mathcal{D}_{1}}\left(\left(\Lambda_{1}, \mathcal{E}, N^{1}\right),\left(\Lambda_{2}, \mathcal{E}, N^{2}\right)\right) \leq 3 n \widetilde{\mathcal{D}_{1}}\left(\left(\Lambda_{1}, \mathcal{E}, N^{1}\right),\left(\Lambda_{3}, \mathcal{E}, N^{3}\right)\right)+3 n \widetilde{\mathcal{D}_{1}}\left(\left(\Lambda_{3}, \mathcal{E}, N^{3}\right),\left(\Lambda_{2}, \mathcal{E}, N^{2}\right)\right)$. Thus,

$$
\widetilde{\mathcal{D}_{1}}\left(\left(\Lambda_{1}, \mathcal{E}, N^{1}\right),\left(\Lambda_{2}, \mathcal{E}, N^{2}\right)\right) \leq \widetilde{\mathcal{D}_{1}}\left(\left(\Lambda_{1}, \mathcal{E}, N^{1}\right),\left(\Lambda_{3}, \mathcal{E}, N^{3}\right)\right)+\widetilde{\mathcal{D}_{1}}\left(\left(\Lambda_{3}, \mathcal{E}, N^{3}\right),\left(\Lambda_{2}, \mathcal{E}, N^{2}\right)\right) .
$$

Proposition 4.3. Let $\left(\Lambda_{1}, \mathcal{E}, N^{1}\right),\left(\Lambda_{2}, \mathcal{E}, N^{2}\right)$ and $\left(\Lambda_{3}, \mathcal{E}, N^{3}\right)$ be three $N N$-SSs over $\mathcal{U}$. If $\left(\Lambda_{1}, \mathcal{E}, N^{1}\right) \sqsubseteq\left(\Lambda_{2}, \mathcal{E}, N^{2}\right) \sqsubseteq$ $\left(\Lambda_{3}, \mathcal{E}, N^{3}\right)$, then

$$
\widetilde{\mathcal{D}_{k}}\left(\left(\Lambda_{1}, \mathcal{E}, N^{1}\right),\left(\Lambda_{2}, \mathcal{E}, N^{2}\right)\right) \leq \widetilde{\mathcal{D}_{k}}\left(\left(\Lambda_{1}, \mathcal{E}, N^{1}\right),\left(\Lambda_{3}, \mathcal{E}, N^{3}\right)\right)
$$

and

for $k=1,2,3,4$.

$$
\widetilde{\mathcal{D}_{k}}\left(\left(\Lambda_{2}, \mathcal{E}, N^{2}\right),\left(\Lambda_{3}, \mathcal{E}, N^{3}\right)\right) \leq \widetilde{\mathcal{D}_{k}}\left(\left(\Lambda_{1}, \mathcal{E}, N^{1}\right),\left(\Lambda_{3}, \mathcal{E}, N^{3}\right)\right)
$$

Proof. We only prove for $k=1$, as the proofs are similar for the others.

Since $\left(\Lambda_{1}, \mathcal{E}, N^{1}\right) \sqsubseteq\left(\Lambda_{2}, \mathcal{E}, N^{2}\right) \sqsubseteq\left(\Lambda_{3}, \mathcal{E}, N^{3}\right)$ is given, the following inequalities are provided from Definition 2.9 for each $x_{l} \in \mathcal{E}$ and $u_{k} \in \mathcal{U}$,

Therefore, we have

$$
\begin{aligned}
& {\left[t_{\Lambda_{1 x}}^{1}\right]^{k} \leq\left[t_{\Lambda_{2 x}}^{2}\right]^{k} \leq\left[t_{\Lambda_{3 x}}^{3}\right]^{k},} \\
& {\left[i_{\Lambda_{1 x}}^{1}\right]^{k} \geq\left[i_{\Lambda_{2 x}}^{2}\right]^{k} \geq\left[i_{\Lambda_{3 x}}^{3}\right]^{k},} \\
& {\left[f_{\Lambda_{1 x}}^{1}\right]^{k} \geq\left[f_{\Lambda_{2 x}}^{2}\right]^{k} \geq\left[f_{\Lambda_{3_{x}}}^{3}\right]^{k} .}
\end{aligned}
$$

$$
\begin{gathered}
\left|\left[t_{\Lambda_{1 x}}^{1}\right]^{k}-\left[t_{\Lambda_{2 x}}^{2}\right]^{k}\right| \leq\left|\left[t_{\Lambda_{1 x}}^{1}\right]^{k}-\left[t_{\Lambda_{3 x}}^{3}\right]^{k}\right|,\left|\left[t_{\Lambda_{2 x}}^{2}\right]^{k}-\left[t_{\Lambda_{3 x}}^{3}\right]^{k}\right| \leq\left|\left[t_{\Lambda_{1 x}}^{1}\right]^{k}-\left[t_{\Lambda_{3 x}}^{3}\right]^{k}\right|, \\
\left|\left[i_{\Lambda_{1 x}}^{1}\right]^{k}-\left[i_{\Lambda_{2 x}}^{2}\right]^{k}\right| \leq\left|\left[i_{\Lambda_{1 x}}^{1}\right]^{k}-\left[i_{\Lambda_{3 x}}^{3}\right]^{k}\right|,\left|\left[i_{\Lambda_{2 x}}^{2}\right]^{k}-\left[i_{\Lambda_{3 x}}^{3}\right]^{k}\right| \leq\left|\left[i_{\Lambda_{1 x}}^{1}\right]^{k}-\left[i_{\Lambda_{3 x}}^{3}\right]^{k}\right|, \\
\left|\left[f_{\Lambda_{1 x}}^{1}\right]^{k}-\left[f_{\Lambda_{2 x}}^{2}\right]^{k}\right| \leq\left|\left[f_{\Lambda_{1 x}}^{1}\right]^{k}-\left[f_{\Lambda_{3 x}}^{3}\right]^{k}\right|,\left|\left[f_{\Lambda_{2 x}}^{2}\right]^{k}-\left[f_{\Lambda_{3 x}}^{3}\right]^{k}\right| \leq\left|\left[f_{\Lambda_{1 x}}^{1}\right]^{k}-\left[f_{\Lambda_{3 x}}^{3}\right]^{k}\right| .
\end{gathered}
$$

Hence

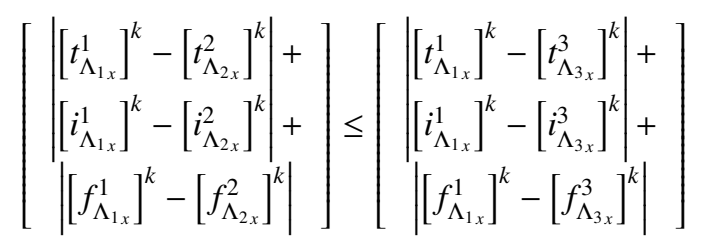

and

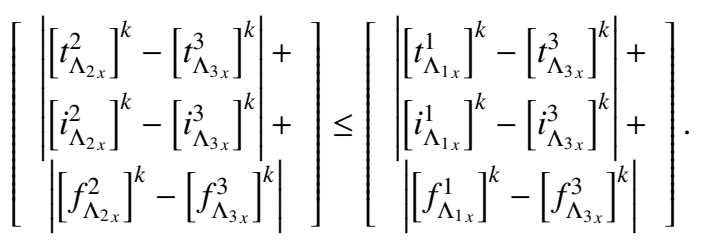


Thus,

$$
\widetilde{\mathcal{D}_{k}}\left(\left(\Lambda_{1}, \mathcal{E}, N^{1}\right),\left(\Lambda_{2}, \mathcal{E}, N^{2}\right)\right) \leq \widetilde{\mathcal{D}_{k}}\left(\left(\Lambda_{1}, \mathcal{E}, N^{1}\right),\left(\Lambda_{3}, \mathcal{E}, N^{3}\right)\right)
$$

and

$$
\widetilde{\mathcal{D}_{k}}\left(\left(\Lambda_{2}, \mathcal{E}, N^{2}\right),\left(\Lambda_{3}, \mathcal{E}, N^{3}\right)\right) \leq \widetilde{\mathcal{D}_{k}}\left(\left(\Lambda_{1}, \mathcal{E}, N^{1}\right),\left(\Lambda_{3}, \mathcal{E}, N^{3}\right)\right)
$$

are proved for $k=1,2,3,4$.

For the similarity measurements, we used the distance measures suggested in our study. Similarity measures $\widetilde{\mathcal{S}_{1}}\left(\left(\Lambda_{1}, \mathcal{E}, N^{1}\right),\left(\Lambda_{2}, \mathcal{E}, N^{2}\right)\right)$ and $\widetilde{\mathcal{S}_{2}}\left(\left(\Lambda_{1}, \mathcal{E}, N^{1}\right),\left(\Lambda_{2}, \mathcal{E}, N^{2}\right)\right)$ between NN-SSs $\left(\Lambda_{1}, \mathcal{E}, N^{1}\right)$ and $\left(\Lambda_{2}, \mathcal{E}, N^{2}\right)$ are given below for $N^{1} \geq N^{2}$,

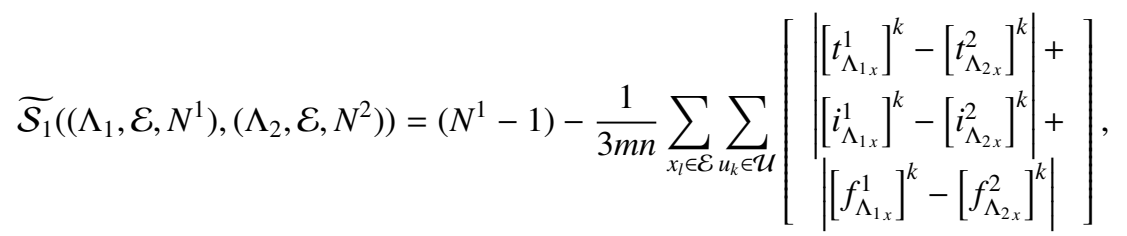

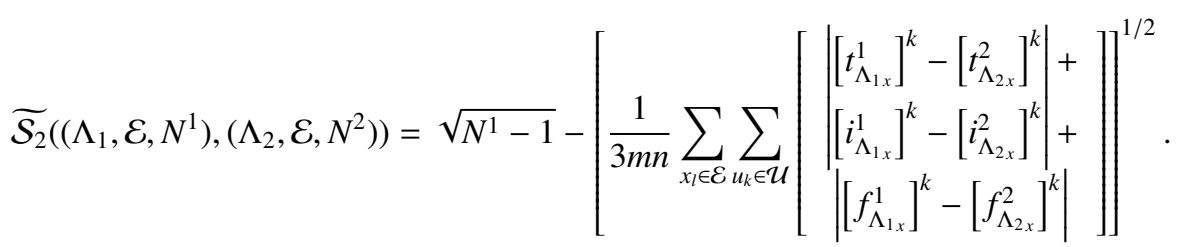

Many of the features we provide for distance measurements can be preserved within similarity measurements, as we create similarity measurements using the proposed distance measurements.

Proposition 4.4. The above defined similarity measurements $\widetilde{\mathcal{S}_{1}}$ and $\widetilde{\mathcal{S}_{2}}$ between $N N$-SSs $\left(\Lambda_{1}, \mathcal{E}, N^{1}\right),\left(\Lambda_{2}, \mathcal{E}, N^{2}\right)$ and $\left(\Lambda_{3}, \mathcal{E}, N^{3}\right)$ satisfy the following properties $\left(\widetilde{\mathcal{S}_{1}}-1, \widetilde{\mathcal{S}_{1}}-4\right)$ for $N^{1} \geq N^{2} \geq N^{3}$,

$\left(\widetilde{\mathcal{S}_{1}}-1\right) 0 \leq \widetilde{\mathcal{S}_{1}}\left(\left(\Lambda_{1}, \mathcal{E}, N^{1}\right),\left(\Lambda_{2}, \mathcal{E}, N^{2}\right)\right) \leq N^{1}-1$ and $0 \leq \widetilde{\mathcal{S}_{2}}\left(\left(\Lambda_{1}, \mathcal{E}, N^{1}\right),\left(\Lambda_{2}, \mathcal{E}, N^{2}\right)\right) \leq \sqrt{N^{1}-1}$.

$\left(\widetilde{\mathcal{S}_{1}}-2\right) \widetilde{\mathcal{S}_{1}}\left(\left(\Lambda_{1}, \mathcal{E}, N^{1}\right),\left(\Lambda_{2}, \mathcal{E}, N^{2}\right)\right)=N^{1}-1$ iff $\left(\Lambda_{1}, \mathcal{E}, N^{1}\right)=\left(\Lambda_{2}, \mathcal{E}, N^{2}\right)$ and $\widetilde{\mathcal{S}_{1}}\left(\left(\Lambda_{1}, \mathcal{E}, N^{1}\right),\left(\Lambda_{2}, \mathcal{E}, N^{2}\right)\right)=\sqrt{N^{1}-1}$ iff $\left(\Lambda_{1}, \mathcal{E}, N^{1}\right)=\left(\Lambda_{3}, \mathcal{E}, N^{3}\right)$.

$\left(\widetilde{\mathcal{S}_{1}}-3\right) \widetilde{\mathcal{S}}_{t}\left(\left(\Lambda_{1}, \mathcal{E}, N^{1}\right),\left(\Lambda_{2}, \mathcal{E}, N^{2}\right)\right)=\widetilde{\mathcal{S}_{1}}\left(\left(\Lambda_{2}, \mathcal{E}, N^{2}\right),\left(\Lambda_{1}, \mathcal{E}, N^{1}\right)\right)$ for $t=1,2$.

$\left(\widetilde{\mathcal{S}_{1}}-4\right)$ If $\left(\Lambda_{1}, \mathcal{E}, N^{1}\right) \sqsubseteq\left(\Lambda_{2}, \mathcal{E}, N^{2}\right) \sqsubseteq\left(\Lambda_{3}, \mathcal{E}, N^{3}\right)$, then $\widetilde{\mathcal{S}_{t}}\left(\left(\Lambda_{1}, \mathcal{E}, N^{1}\right),\left(\Lambda_{3}, \mathcal{E}, N^{3}\right)\right) \leq \widetilde{\mathcal{S}_{1}}\left(\left(\Lambda_{2}, \mathcal{E}, N^{2}\right),\left(\Lambda_{1}, \mathcal{E}, N^{1}\right)\right)$ and $\widetilde{\mathcal{S}}_{t}\left(\left(\Lambda_{1}, \mathcal{E}, N^{1}\right),\left(\Lambda_{3}, \mathcal{E}, N^{3}\right)\right) \leq \widetilde{\mathcal{S}_{1}}\left(\left(\Lambda_{2}, \mathcal{E}, N^{2}\right),\left(\Lambda_{3}, \mathcal{E}, N^{3}\right)\right)$ for $t=1,2$.

Proof. The proof is clear from Theorem 4.2 and Proposition 4.3.

Definition 4.5. The two NN-SSs $\left(\Lambda_{1}, \mathcal{E}, N^{1}\right)$ and $\left(\Lambda_{2}, \mathcal{E}, N^{2}\right)$ are $\lambda$ similar if and only if $\widetilde{\mathcal{S}_{1}}\left(\left(\Lambda_{1}, \mathcal{E}, N^{1}\right),\left(\Lambda_{2}, \mathcal{E}, N^{2}\right)\right) \geq \lambda$ for $N^{1} \geq N^{2}$, i.e.,

$$
\left(\Lambda_{1}, \mathcal{E}, N^{1}\right) \approx^{\lambda}\left(\Lambda_{2}, \mathcal{E}, N^{2}\right) \Leftrightarrow \widetilde{\mathcal{S}_{1}}\left(\left(\Lambda_{1}, \mathcal{E}, N^{1}\right),\left(\Lambda_{2}, \mathcal{E}, N^{2}\right)\right) \geq \lambda, \quad \lambda \in\left[0,\left(N^{1}-1\right)\right] .
$$

$\left(\Lambda_{1}, \mathcal{E}, N^{1}\right)$ and $\left(\Lambda_{2}, \mathcal{E}, N^{2}\right)$ are significantly similar if $\widetilde{\mathcal{S}_{1}}\left(\left(\Lambda_{1}, \mathcal{E}, N^{1}\right),\left(\Lambda_{2}, \mathcal{E}, N^{2}\right)\right) \geq\left(N^{1}-1\right) / 2$.

Definition 4.6. The two NN-SSs $\left(\Lambda_{1}, \mathcal{E}, N^{1}\right)$ and $\left(\Lambda_{2}, \mathcal{E}, N^{2}\right)$ are $\lambda$ similar if and only if $\widetilde{\mathcal{S}_{2}}\left(\left(\Lambda_{1}, \mathcal{E}, N^{1}\right),\left(\Lambda_{2}, \mathcal{E}, N^{2}\right)\right) \geq \lambda$ for $N^{1} \geq N^{2}$, i.e.,

$$
\left(\Lambda_{1}, \mathcal{E}, N^{1}\right) \approx^{\lambda}\left(\Lambda_{2}, \mathcal{E}, N^{2}\right) \Leftrightarrow \widetilde{\mathcal{S}_{2}}\left(\left(\Lambda_{1}, \mathcal{E}, N^{1}\right),\left(\Lambda_{2}, \mathcal{E}, N^{2}\right)\right) \geq \lambda, \quad \lambda \in\left[0, \sqrt{N^{1}-1}\right] .
$$

$\left(\Lambda_{1}, \mathcal{E}, N^{1}\right)$ and $\left(\Lambda_{2}, \mathcal{E}, N^{2}\right)$ are significantly similar if $\widetilde{\mathcal{S}_{2}}\left(\left(\Lambda_{1}, \mathcal{E}, N^{1}\right),\left(\Lambda_{2}, \mathcal{E}, N^{2}\right)\right) \geq \sqrt{N^{1}-1} / 2$. 


\section{Application of Similarity Measurements for NN-SS}

In this section, applying the similarity measures to decision making, we present a handling method for the decisionmaking problem by means of the similarity measures between NN-SSs.

In the following example, we will try to estimate the most appropriate staff for the conditions expressed by a company. For this, we first construct a model NN-SS expressed by the company for the most appropriate staff and the NN-SS for candidate staff. Then, we calculated the similarity measurements for the built NN-SSs. If the NN-SSs are significantly similar, then we decide that the staff taken into evaluation will possibly be hired.

Now we consider a universal set $\mathcal{U}=\{y=Y E S, n=N O\}$ and the set of parameters $\mathcal{E}=\left\{x_{1}=\right.$ determination to work, $x_{2}=$ foreign language proficiency, $x_{3}=$ hardworking $\}$ is the set containing the characteristics of the staff desired by the company. Our model NN-SS for the most suitable staff $(\Omega, \mathcal{E}, 8)$ is given below and this can be prepared with the help of a expert person:

$$
(\Omega, \mathcal{E}, 8)=\left\{\begin{array}{c}
\left(x_{1},(y,\langle 6,5,7\rangle),(n,\langle 6,7,5\rangle)\right), \\
\left(x_{2},(y,\langle 6,7,6\rangle),(n,\langle 6,7,7\rangle)\right), \\
\left(x_{1},(y,\langle 7,6,5\rangle),(n,\langle 6,5,7\rangle)\right)
\end{array}\right\} .
$$

Now, the three candidates applying to this company will be evaluated. The first candidate $(\mathrm{C} 1)$ is represented by $\left(\Omega_{1}, \mathcal{E}, 6\right)$ given below,

$$
\left(\Omega_{1}, \mathcal{E}, 6\right)=\left\{\begin{array}{c}
\left(x_{1},(y,\langle 5,5,4\rangle),(n,\langle 5,4,5\rangle)\right), \\
\left(x_{2},(y,\langle 4,3,5\rangle),(n,\langle 4,5,5\rangle)\right), \\
\left(x_{1},(y,\langle 4,5,4\rangle),(n,\langle 5,5,4\rangle)\right)
\end{array}\right\} .
$$

Then we find the similarity measures of these two sets as:

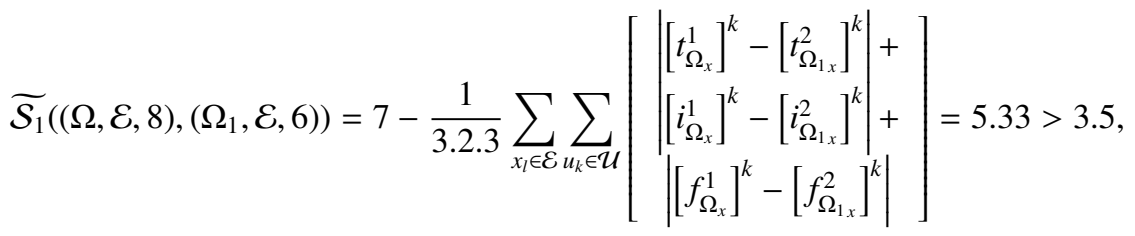

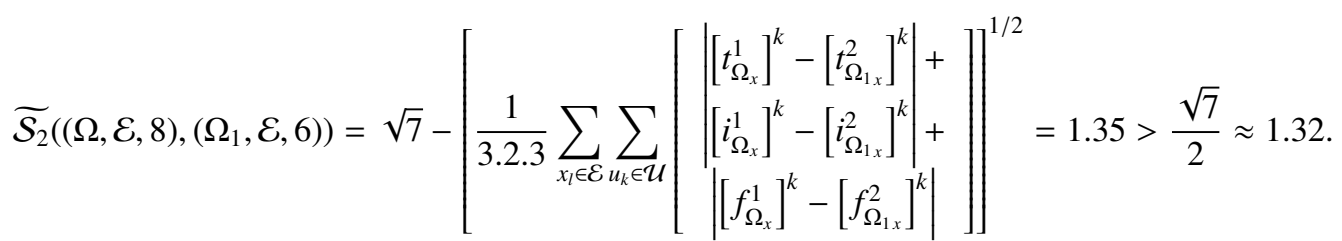

Hence the two NN-SSs are significantly similar. Therefore, we conclude that hiring the C1-candidate may be correct. The C2-candidate with the following characteristics whose corresponding NN-SS $\left(\Omega_{2}, \mathcal{E}, 7\right)$ is given below:

$$
\left(\Omega_{2}, \mathcal{E}, 7\right)=\left\{\begin{array}{c}
\left(x_{1},(y,\langle 2,6,3\rangle),(n,\langle 1,2,2\rangle)\right), \\
\left(x_{2},(y,\langle 2,1,4\rangle),(n,\langle 3,6,2\rangle)\right), \\
\left(x_{1},(y,\langle 1,0,2\rangle),(n,\langle 3,3,6\rangle)\right)
\end{array}\right\} .
$$

Then $\widetilde{\mathcal{S}_{1}}\left((\Omega, \mathcal{E}, 8),\left(\Omega_{2}, \mathcal{E}, 7\right)\right)=3.44<3.5$ and $\widetilde{\mathcal{S}_{1}}\left((\Omega, \mathcal{E}, 8),\left(\Omega_{2}, \mathcal{E}, 7\right)\right)=0.76<1.32$. Here the two NN-SSs are not significantly similar. Therefore, we conclude that hiring the $\mathrm{C} 1$-candidate may not be correct. Finally, the $\mathrm{C} 3$-candidate with the following characteristics whose corresponding NN-SS $\left(\Omega_{3}, \mathcal{E}, 7\right)$ is given below:

$$
\left(\Omega_{3}, \mathcal{E}, 7\right)=\left\{\begin{array}{c}
\left(x_{1},(y,\langle 6,4,2\rangle),(n,\langle 4,1,6\rangle)\right), \\
\left(x_{2},(y,\langle 5,2,4\rangle),(n,\langle 3,4,2\rangle)\right), \\
\left(x_{1},(y,\langle 6,3,5\rangle),(n,\langle 1,3,6\rangle)\right)
\end{array}\right\} .
$$

Then $\widetilde{\mathcal{S}_{1}}\left((\Omega, \mathcal{E}, 8),\left(\Omega_{3}, \mathcal{E}, 7\right)\right)=4.44>3.5$ and $\widetilde{\mathcal{S}_{1}}\left((\Omega, \mathcal{E}, 8),\left(\Omega_{3}, \mathcal{E}, 7\right)\right)=1.04<1.32$. It is difficult to decide in such cases. However, it may be useful to take advantage of another similarity measurement. Since each similarity measurement may have different advantages, it may not be correct to do a ranking of superiority among them. 


\section{Conclusion}

This paper discusses N-soft sets, which is a generalized mathematical model of soft sets under the framework of neutrosophic logic, and brings neutrosophic N-soft sets to the literature. With this new model taking neutrosphic logic and soft sets that attract the attention of many researchers today, it offers a more ideal approach to uncertainty. Moreover, a section has been created that examines some properties related to this concept. Then, some products and useful operations on the neutrosophic $\mathrm{N}$-soft sets are derived. In addition, we have given the similarity measures of the neutrosophic N-soft sets by defining the Euclidean and Hamming distance measures between two neutrosophic N-soft sets. Finally, an application is presented on how to take advantage of the similarity measures defined for a decision making problem.

The researchers who will benefit from this paper in the future may be able to achieve more impressive results in applying different mathematical models to their decision making problems regarding uncertainty situations. Thanks to the success of neutrosophic logic and N-soft set especially in the area of uncertainty, we think that neutrosophic N-soft set we are working on will be an important research contribution.

\section{CONFLICTS OF INTEREST}

The authors declare that there are no conflicts of interest regarding the publication of this article.

\section{Authors Contribution Statement}

All authors have contributed sufficiently in the planning, execution, or analysis of this study to be included as authors. All authors have read and agreed to the published version of the manuscript.

\section{REFERENCES}

[1] Abu Qamar, M., Hassan, N., An approach toward a Q-neutrosophic soft set and its application in decision making, Symmetry, 11(2)(2019), 139.

[2] Akram, M., Adeel, A., Alcantud, J.C.R., Fuzzy N-soft sets: a novel model with applications, Journal of Intelligent and Fuzzy Systems, 35(4)(2018), 4757-4771.

[3] Akram, M., Adeel, A., Alcantud, J.C.R., Group decision-making methods based on hesitant N-soft sets, Expert Systems with Applications, 115(2019), 95-105.

[4] Akram, M., Adeel, A., Alcantud, J.C.R., Hesitant fuzzy N-soft sets: A new model with applications in decision-making, Journal of Intelligent and Fuzzy Systems, 36(6)(2019), 6113-6127.

[5] Akram, M., Adeel, A., TOPSIS approach for MAGDM based on interval-valued hesitant fuzzy N-soft environment, International Journal of Fuzzy Systems, 21(3)(2019), 993-1009.

[6] Akram, M., Ali, G., Alcantud, J. C. R., New decision-making hybrid model: intuitionistic fuzzy N-soft rough sets, Soft Computing, 23(20)(2019), 9853-9868.

[7] Ali, M. I., Feng, F., Liu, X., Min, W.K., Shabir, M., On some new operations in soft set theory, Computers and Mathematics with Applications, 57(2007), 1547-1553.

[8] Alkhazaleh, S., Salleh, A.R., Hassan, N., Fuzzy parameterized interval-valued fuzzy soft set, Applied Mathematical Sciences, 5(2011), 33353346.

[9] Alkhazaleh, S., Salleh, A.R., Soft expert sets, Advances in Decision Sciences, 15(2011), 757868-1.

[10] Atanassov, K., Intuitionistic fuzzy sets, Fuzzy Sets Systems, 20(1986), 87-96.

[11] Atanassov, K., Gargov, G., Interval valued intuitionistic fuzzy sets, Fuzzy Set Systems, 31(1989), 343-349.

[12] Broumi, S., Generalized neutrosophic soft set, International Journal of Computer Science, Engineering and Information Technology, 3(2)(2013), 17-30.

[13] Broumi, S., Smarandache, F., Intuitionistic neutrosophic soft set, Journal of Information and Computing Science, 8(2)(2013), $130-140$.

[14] Broumi, S., Deli, I., Smarandache, F., $N$-valued interval neutrosophic sets and their application in medical diagnosis, Critical Review, Center for Mathematics of Uncertainty, Creighton University, USA, 10(2015), 46-69.

[15] Broumi, S., Deli, I., Correlation measure for neutrosophic refined sets and its application in medical diagnosi, Palestine Journal of Mathematics, 5(1)(2016), 135-143.

[16] Chen, D.G., Tsang, E.C.C., Yeung, D.S., Xizhao, W., The parameterization reduction of soft sets and its applications, Computers and Mathematics with Applications, 49(2005), 757-763.

[17] Çagman, N., Enginoglu, S., Soft set theory and uni-int decision making, European Journal of Operational Research, 207(2010), 848-855.

[18] Dalkılıç, O., Demirtas N., Bipolar soft filter, Journal of Universal Mathematics, 3(1)(2020), 21-27.

[19] Dalkılıç, O., Demirtaş, N., VFP-soft sets and its application on decision making problems, Journal of Polytechnic, (2021), https://doi.org/10.2339/politeknik.685634. 
[20] Dalkılıç, O., An application of VFPFSS's in decision making problems, Journal of Polytechnic, (2021), https://doi.org/10.2339/politeknik.758474.

[21] Das, S., Kar, D.S., Group decision making in medical system: an intuitionistic fuzzy soft set approach, Applied Soft Computing, 24(2014), 196-211.

[22] Deli, I., Interval-valued neutrosophic soft sets and its decision making, International Journal of Machine Learning and Cybernetics, 8(2)(2017), 665-676.

[23] Deli, I., Subas, Y., A ranking method of single valued neutrosophic numbers and its applications to multi-attribute decision making problems, International Journal of Machine Learning and Cybernetics, 8(4)(2017), 1309-1322.

[24] Demirtaş, N., Dalkılıç, O., An application in the diagnosis of prostate cancer with the help of bipolar soft rough sets, on Mathematics and Mathematics Education (ICMME 2019), KONYA, (2019), 283.

[25] Demirtas, N., Hussain, S., Dalkılıç, O., New approaches of inverse soft rough sets and their applications in a decision making problem, Journal of Applied Mathematics and Informatics, 38(3-4)(2020), 335-349.

[26] Demirtaş, N., Dalkılıç, O., Decompositions of soft $\alpha$-continuity and soft A-continuity, Journal of New Theory, 31(2020), 86-94.

[27] Fatimah, F., Rosadi, D., Hakim, R.B.F., Alcantud, J.C.R., N-soft sets and their decision-making algoritms, Soft Computing, 22(2018), 38293842.

[28] Feng, F., Jun, Y.B., Liu, X., Li, L., An adjustable approach to fuzzy soft sets based decision making, Journal of Computational and Applied Mathematics, 234(2010), 10-20.

[29] Feng, F., Li, C., Davvaz, B., Irfan Ali, M., Soft sets combined with fuzzy sets and rough sets: a tentative approach, Soft Computing, 14(2010), 899-911.

[30] Grattan-Guiness, I., Fuzzy membership mapped onto interval and many-valued quantities, Z Math Logic Grundlagen Mathematics, 22(1975), 149-160.

[31] Jahn, K.U., Intervall-wertige Mengen, Mathematische Nachrichten, 68(1975), 115-132.

[32] Jiang, Y., Tang, Y., Chen, Q., An adjustable approach to intuitionistic fuzzy soft sets based decision making, Applied Mathematical Modelling, 35(2011), 824-836.

[33] Kamal, N.L.A.M., Abdullah, L., Abdullah, I., Alkhazaleh, S., Karaaslan, F., Multivalued interval neutrosophic soft set: formulation and theory, Neutrosophic Sets and Systems, 30(1)(2019), 12.

[34] Kong, Z., Gao, L.Q., Wang, L.F., Li, S., The normal parameter reduction of soft sets and its algorithm, Computers and Mathematics with Applications, 56(2008), 3029-3037.

[35] Maji, P.K., Biswas, R., Roy, A.R., Fuzzy soft set theory, Journal of Fuzzy Mathematics, 9(3)(2001), 589-602.

[36] Maji, P.K., Roy, A.R., Biswas, R., An application of soft sets in a decision making problem, Computers and Mathematics with Applications, 44(2002), 1077-1083.

[37] Maji, P.K., Roy, A.R., Biswas, R., Soft set theory, Computers and Mathematics with Applications, 24(2003), 555-562.

[38] Maji, P.K., Roy, A.R., Biswas, R., On intuitionistic fuzzy soft sets, Journal of Fuzzy Mathematics, 12(3)(2004), 669-683.

[39] Maji, P.K., A neutrosophic soft set approach to a decision making problem, Annals of Fuzzy Mathematics and Informatics, 3(2)(2012), 313319.

[40] Maji, P.K., Neutrosophic soft set, Computers and Mathematics with Applications, 45(2013), 555-562.

[41] Molodtsov, D., Soft set theory-first results, Computers and Mathematics with Applications, 37(1999), $19-31$.

[42] Pawlak, Z., Rough sets, International Journal of Computer and Information Sciences, 11(1982), 341-356.

[43] Qin, K., Hong, Z., On soft equality, J. Computers and Mathematics with Applications, 234(5)(2010), 1347-1355.

[44] Riaz, M., Çagman, N., Zareef, I., Aslam, M., N-soft topology and its applications to multi-criteria group decision making,Journal of Intelligent and Fuzzy Systems, 36(6)(2019), 6521-6536.

[45] Saha, A., Broumi, S., Parameter reduction of neutrosophic soft sets and their applications, Neutrosophic Sets and Systems, 32(1)(2020), 1.

[46] Sambuc, R., Fonctions '-floues: Application l'aide au diagnostic en pathologie thyroidienne. Ph. D. Thesis Univ. Marseille, France, (1975).

[47] Smarandache, F., A Unifying Field in Logics. Neutrosophy: Neutrosophic Probability, Set and Logic, American Research Press, Rehoboth, 1999.

[48] Smarandache, F., Neutrosophy a new branch of philosophy, Multi. Val. Logic-Special Issue: Neutrosophy and Neutrosophic Logic, 8(2002), 297-384.

[49] Smarandache, F., Neutrosophic set, a generalisation of the intuitionistic fuzzy sets, Indian Journal of Pure and Applied Mathematics, 24(2005), 287-297.

[50] Uluçay, V., Sahin, M., Hassan, N., Generalized neutrosophic soft expert set for multiple-criteria decision-making, Symmetry, 10(10)(2018), 437.

[51] Wang, H., Smarandache, F., Zhang, Y.Q., Sunderraman, R., Single valued neutrosophic sets, Multispace Multistruct, 4(2010), 410-413.

[52] Zadeh, L.A., Fuzzy sets, Information and Control, 8(1965), 338-353.

[53] Zadeh, L., The concept of a linguistic variable and its application to approximate reasoning-I, Information Sciences, 8(1975), $199-249$. 